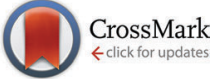

Cite this: New J. Chem., 2016, 40,245

Received (in Montpellier, France) 8th September 2015

Accepted 23rd October 2015

DOI: $10.1039 / c 5 n j 02406 d$

www.rsc.org/njc

\section{Novel indenyl ligands bearing electron-withdrawing functional groups $\dagger$}

\author{
Iva Honzíčková, ${ }^{a}$ Jaromír Vinklárek, ${ }^{a}$ Carlos C. Romão, ${ }^{b}$ Zdeňka Růžičkováa and \\ Jan Honzíček*c
}

\begin{abstract}
A series of molybdenum complexes bearing new ligands is reported. The study covers a series of molybdenum compounds with the $\eta^{5}$-coordinated indenyl ligand substituted with acyl-, ester- and amide-functions. This portfolio was extended by adding one representative with a $\eta^{3}$-coordinated estersubstituted indenyl ligand. The functionalized indenes, necessary for the assembly, were prepared by convenient routes starting from inexpensive and readily available materials, enabling their production on a multigram scale. All structural types presented in this experimental study were supported by $\mathrm{X}$-ray crystallographic data.
\end{abstract}

\section{Introduction}

The attachment of functional groups at cyclopentadienyl (Cp) rings of transition metal complexes can change considerably their electronic properties as well as the range of potential applications. ${ }^{1,2}$ So far, many different substitution patterns have been developed for the $\mathrm{Cp}$ ligand but only a few of them cover electron-withdrawing substituents and are applicable for ligands with extended $\pi$-systems such as indenyl (Ind) or fluorenyl (Flu). These congeners of the $\mathrm{Cp}$ bearing annulated benzene rings show unique properties with consequent implications in the design of new catalytic systems. ${ }^{3}$ Hence, the replacement of the Cp ligand with a larger $\pi$-system often has a strong effect on reactivity. ${ }^{4}$ This so-called "indenyl effect" accelerates the reaction rates or switches the reaction pathway due to a lower energetic barrier of the haptotropic rearrangement of the $\pi$-ligand. ${ }^{5}$ Recent studies have further revealed that a subtle modification of the indenyl ligand (e.g. replacement of one hydrogen with a methyl group) may play a crucial role in activation of coordinated ligands. ${ }^{2,6,7}$

The modification of the indenyl ligand with electron-withdrawing substituents is rather rare. In 2000, Deck et al. extended a synthetic route giving perfluoroaryl-functionalized cyclopentadienides ${ }^{8}$ on the indenes and described their rhenium complexes. ${ }^{9}$ Some other strong

\footnotetext{
${ }^{a}$ Department of General and Inorganic Chemistry, Faculty of Chemical Technology, University of Pardubice, Studentská 573, Pardubice, Czech Republic

${ }^{b}$ Instituto de Tecnologia Química e Biológica António Xavier, Universidade Nova de Lisboa, Av. Da República, 2780-157 Oeiras, Portugal

${ }^{c}$ Institute of Chemistry and Technology of Macromolecular Materials Faculty of

Chemical Technology, University of Pardubice, Studentská 573, Pardubice,

Czech Republic. E-mail: jan.honzicek@upce.cz; Fax: +420 466037068

$\dagger$ CCDC 1416908-1416913. For crystallographic data in CIF or other electronic

format see DOI: $10.1039 / \mathrm{c} 5 \mathrm{nj} 02406 \mathrm{~d}$
}

electron-withdrawing substituents such as $-\mathrm{F},{ }^{10}-\mathrm{CN},{ }^{11}-\mathrm{COOR},{ }^{12,13}$ $-\mathrm{CONHR}^{14,15}$ and $-\mathrm{COR},{ }^{11,12,16}$ have been successfully attached on the $\mathrm{Cp}$ ring of various transition metal complexes using different strategies. However, the synthesis of the indenyl congeners has not been reported, although some of the suitable indene precursors are already known for several decades. ${ }^{17}$

The aim of this study is to extend a family of indenyl ligands with ester-, amide- and acyl-functionalized derivatives. It will cover a series of modified indenes and unprecedented indenyl complexes. It is expected that a strong electron-withdrawing power of the attached functional groups will lead to more electron deficient metal centers that should be beneficial for their future application in catalysis. We decided to start our investigations with a familiar and well defined fragment, $\left(\eta^{3}-\mathrm{C}_{3} \mathrm{H}_{5}\right) \mathrm{Mo}(\mathrm{CO})_{2}$, before moving into unexplored areas. The allyl molybdenum scaffold is accessible from the halide precursor $\left[\left(\eta^{3}-\mathrm{C}_{3} \mathrm{H}_{5}\right) \mathrm{Mo}(\mathrm{CO})_{2}(\mathrm{NCMe})_{2} \mathrm{X}\right]$, which is an excellent starting material for the incorporation of a monoanionic $\pi$-ligand into the molybdenum coordination sphere. ${ }^{15,16,18-20}$

\section{Results and discussion}

\section{Ester-functionalized indenes}

Three approaches for the synthesis of ester-functionalized indenes were examined. The obvious one using the reaction of sodium indenide with one equivalent of chloroformate was found to be ineffective since the 1,1-disubstituted derivative appears as a major product. In case of the methyl ester, 3-( $\mathrm{MeOCO}) \mathrm{C}_{9} \mathrm{H}_{7}(2)$ and 1,1-(MeOCO) $)_{2} \mathrm{C}_{9} \mathrm{H}_{6}$ (2a) were isolated in $2 \%$ and $15 \%$ yields, respectively (Scheme 1).

Alternatively, a series of ester-functionalized indenes (3-5) was prepared by an esterification of indene-3-carboxylic acid 


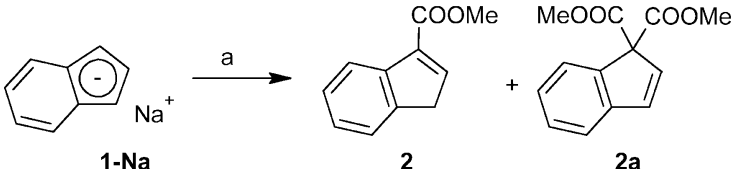

Scheme 1 Reaction of sodium indenide (1-Na) with chloroformate. Reagent: ClCOOMe.

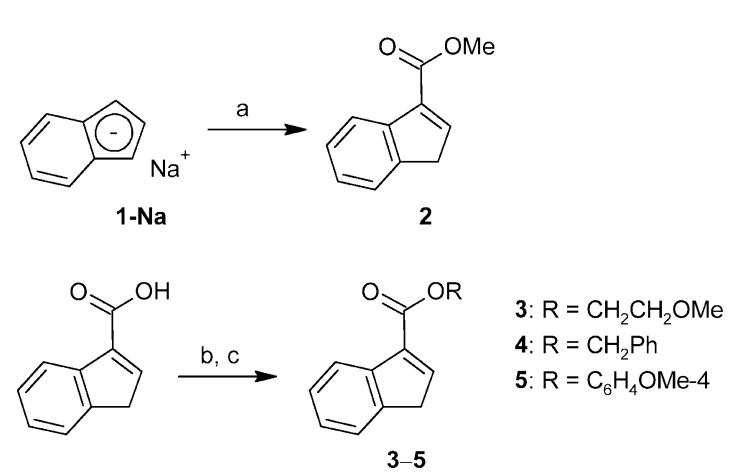

Scheme 2 Synthesis of ester-functionalized indenes. Reagents: (a) $\mathrm{OC}(\mathrm{OMe})_{2}$ (b) $\mathrm{SOCl}_{2}$, (c) $\mathrm{ROH}$.

according to a protocol reported for methyl ester 2, see Scheme $2 .{ }^{21}$ Since availability of the starting $3-(\mathrm{HOCO}) \mathrm{C}_{9} \mathrm{H}_{7}$ is a limiting factor of the procedure, an alternative strategy was used for the preparation of $3-(\mathrm{MeOCO}) \mathrm{C}_{9} \mathrm{H}_{7}$ (2) on a larger scale. The reaction of sodium indenide (1-Na) with dimethyl carbonate gives the functionalized indene 2 in one step and about $41 \%$ isolated yield without the need of a chromatographic purification step (Scheme 2).

\section{Ester-functionalized indenyl molybdenum compounds}

The ability of ester-functionalized indenyl ligands to form $\eta^{5}$-coordination compounds was evidenced for the following molybdenum species. The series of ester-substituted indenes (3-5) was deprotonated with $n$-butyl lithium. The reaction of indenides (3-Li-5-Li) formed in situ with the halide complex $\left[\left(\eta^{3}-\mathrm{C}_{3} \mathrm{H}_{5}\right) \mathrm{Mo}(\mathrm{CO})_{2}(\mathrm{NCMe})_{2} \mathrm{Cl}\right](6)$, accompanied by a loss of the stabilizing $\mathrm{MeCN}$ ligands, resulted in the formation of $\eta^{5}$-indenyl compounds $\left[\left(\eta^{3}-\mathrm{C}_{3} \mathrm{H}_{5}\right)\left\{\eta^{5}-1\right.\right.$-(ROCO $\left.\left.) \mathrm{C}_{9} \mathrm{H}_{6}\right\} \mathrm{Mo}(\mathrm{CO})_{2}\right]$ (7-10), see Scheme 3.

The infrared and Raman spectra of compounds 7-10 show two CO stretching bands in a range typical for terminal carbonyl ligands, see Table 1. The CO stretching bands of the ester groups vary between 1702 and $1722 \mathrm{~cm}^{-1}$, thus revealing a very low
Table 1 Summary of the infrared and Raman data for the ester-functionalized molybdenum complexes ${ }^{a}$

\begin{tabular}{|c|c|c|c|c|}
\hline & \multicolumn{2}{|l|}{ Infrared } & \multicolumn{2}{|l|}{ Raman } \\
\hline & $\nu(\mathrm{C} \equiv \mathrm{O})$ & $\nu(\mathrm{C}=\mathrm{O})$ & $\nu(\mathrm{C} \equiv \mathrm{O})$ & $\nu(\mathrm{C}=\mathrm{O})$ \\
\hline 7 & $\begin{array}{l}1934 \\
1866\end{array}$ & 1714 & $\begin{array}{l}1944 \\
1861\end{array}$ & 1715 \\
\hline 8 & $\begin{array}{l}1947 \\
1869\end{array}$ & 1712 & $-^{b}$ & $-^{b}$ \\
\hline 9 & $\begin{array}{l}1932 \\
1865\end{array}$ & 1702 & $\begin{array}{l}1942 \\
1856\end{array}$ & 1705 \\
\hline 10 & $\begin{array}{l}1944 \\
1865\end{array}$ & 1722 & $\begin{array}{l}1946 \\
1862\end{array}$ & 1714 \\
\hline 12 & $\begin{array}{l}1943 \\
1876 \\
1828\end{array}$ & 1694 & $-^{b}$ & $-^{b}$ \\
\hline
\end{tabular}

${ }^{a}$ The stretching frequencies are given in $\mathrm{cm}^{-1} .{ }^{b}$ The Raman spectrum was not obtained owing to luminescence.

delocalization of $\pi$-electrons of the $\mathrm{C}=\mathrm{O}$ group and excluding an alternative $\kappa$ - $O$-coordination mode of the functionalized indenyl ligand. The ${ }^{1} \mathrm{H}$ NMR spectra of compounds $\mathbf{7 - 1 0}$ show the presence of two conformers arising from a different orientation of the $\eta^{3}$-bonded allyl ligand. The signals of the allyl ligand were assigned to a conformer with the allyl ligand eclipsed with OC-Mo-CO (exo) and that with a staggered conformation (endo) according to data published for the unsubstituted analogue. ${ }^{18}$ At room temperature, the exo-conformer predominates over the endo-conformer. The molar ratio (exo/endo) was found to be $3: 1$ for compounds 7-10 correlating well with data published for monosubstituted derivatives of $\left[\left(\eta^{3}-\mathrm{C}_{3} \mathrm{H}_{5}\right)\left(\eta^{5}\right.\right.$-Ind $\left.) \mathrm{Mo}(\mathrm{CO})_{2}\right] .{ }^{7}$

The solid state structure of compound 7 was determined by the single crystal X-ray analysis. The molecule has a distorted tetrahedral structure with allyl, indenyl and two carbonyl ligands around molybdenum in the formal oxidation state II, see Fig. 1. The geometric parameters describing the coordination sphere of molybdenum are listed in Table 2. The $\eta^{3}$-coordinated allyl ligand is positionaly disordered and splits into exo and endo conformations with an occupancy of about $7: 3$. The substituted indenyl ligand is $\eta^{5}$-coordinated as evidenced by a low value of the envelope fold angle $\left[\Omega=4.5(3)^{\circ}\right]$ and also by $\Delta(\mathrm{M}-\mathrm{C})[0.119(3) \AA]$. The ester group is almost coplanar with the indenyl framework. The dihedral angle between the $\mathrm{C}_{5}$ ring of indenyl and a plane defined by atoms $\mathrm{C} 1, \mathrm{C} 10, \mathrm{O} 1$ and $\mathrm{O} 2$ is $1.28(16)^{\circ}$.

Having obtained a series of molybdenum compounds with $\eta^{5}$-bonded ester-substituted indenyl ligands, we sought to extend

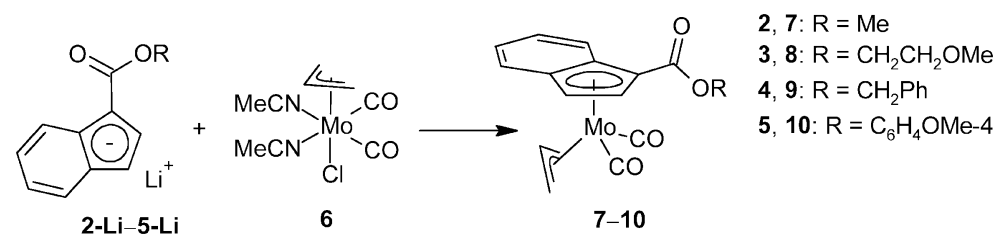

Scheme 3 Synthesis of molybdenum complexes bearing ester-functionalized indenyl. 


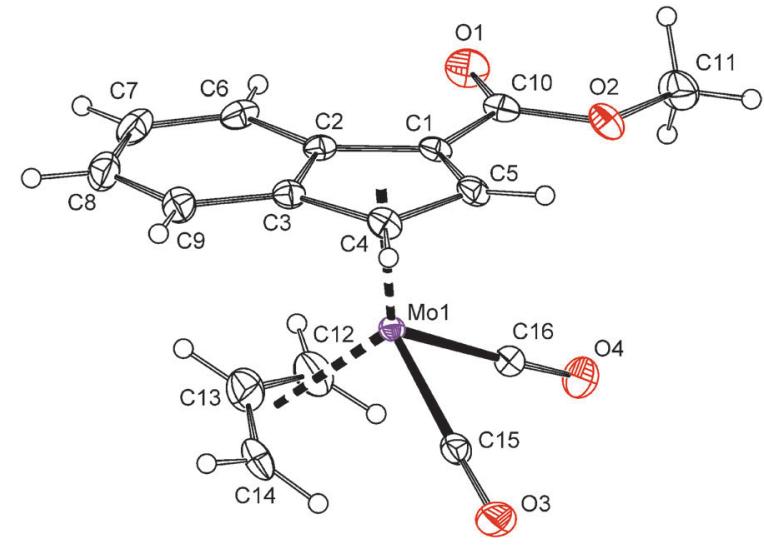

Fig. 1 ORTEP drawing of the molybdenum compound $\left[\left(\eta^{3}-\mathrm{C}_{3} \mathrm{H}_{5}\right)\left\{\eta^{5}-1-\right.\right.$ $\left.(\mathrm{MeOCO}) \mathrm{C}_{9} \mathrm{H}_{6}\right\} \mathrm{Mo}(\mathrm{CO})_{2}$ ] present in the crystal structure of 7 . Thermal ellipsoids are drawn at the $30 \%$ probability level. Only the major conformation of the positionaly disordered allyl ligand is shown for clarity.

the portfolio of such compounds by (i) adding a representative with a $\eta^{3}$-coordinated ester-substituted indenyl ligand, (ii) introducing an acetyl substituent as a representative of a stronger electron-withdrawing substituent and (iii) developing a versatile route for amide-functionalized derivatives.

On the first count, we chose the mixed-indenyl species of type $\left[\left(\eta^{3}\right.\right.$-Ind $)\left(\eta^{5}\right.$-Ind $\left.) \mathrm{Mo}(\mathrm{CO})_{2}\right]$. The methyl ester derivative $\left[\left\{\eta^{3}-1-(\mathrm{MeOCO}) \mathrm{C}_{9} \mathrm{H}_{6}\right\}\left(\eta^{5}\right.\right.$-Ind) $\left.\mathrm{Mo}(\mathrm{CO})_{2}\right](12)$ was prepared by a reaction of $2-\mathbf{L i}$ with one equivalent of $\left[\left\{\left(\eta^{5}-\mathrm{Ind}\right) \mathrm{Mo}(\mathrm{CO})_{2}(\mu-\mathrm{Cl})\right\}_{2}\right]$ (11) in a moderate isolated yield (43\%), see Scheme 4 . Coordination of the indenyl ligand was evidenced by infrared spectroscopy, which shows the stretching bands of the carbonyl ligands at frequencies $\left(\nu_{\mathrm{a}}: 1943 \mathrm{~cm}^{-1} ; \nu_{\mathrm{s}}: 1876,1828 \mathrm{~cm}^{-1}\right)$ similar to those reported for the unsubstituted parent compound $\left[\left(\eta^{3}\right.\right.$-Ind)$\left(\eta^{5} \text {-Ind) } \mathrm{Mo}(\mathrm{CO})_{2}\right]^{23}$ The appearance of two bands of the $\nu_{\mathrm{s}}(\mathrm{CO})$ is due to a vibration coupling of the carbonyl ligands in the crystal lattice. The CO stretching band of the ester group appears at a lower frequency $\left(1694 \mathrm{~cm}^{-1}\right)$ than that of compound 7 thus implying a more electrophilic character of the molybdenum center. The ${ }^{1} \mathrm{H}$ NMR spectrum shows broadened signals indicating a fluxional structure in solution due to a fast hapticity

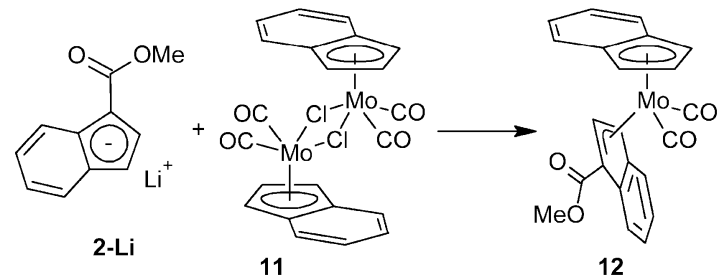

Scheme 4 Synthesis of a mixed indenyl molybdenum complex.

exchange of the indenyl rings. This observation correlates well with the properties of the unsubstituted analogue $\left[\left(\eta^{3}\right.\right.$-Ind)$\left(\eta^{5}\right.$-Ind $\left.) \mathrm{Mo}(\mathrm{CO})_{2}\right]$ that gives only one set of signals for both indenyl ligands. ${ }^{24}$

The X-ray diffraction analysis reveals that the less donating ester-substituted indenyl ligand adopts the $\eta^{3}$-coordination mode in the solid state, whereas the electron richer unsubstituted indenyl stays $\eta^{5}$-coordinated, see Fig. 2 . The hapticity of indenyl ligands is clearly elucidated from the slip parameters listed in Table 2 . Hence, the substituted indenyl has considerably higher values of $\Omega$ and $\Delta(\mathrm{M}-\mathrm{C})$ then the unsubstituted one. Compound 12 adopts a conformation, similar to the unsubstituted counterpart, ${ }^{23}$ with the $\eta^{3}$-indenyl ligand in exo-conformation and the $\mathrm{C}_{6}$-ring of the $\eta^{5}$-indenyl facing away from the carbonyl ligands thereby avoiding repulsive interactions. The ester group is almost coplanar with the $\eta^{3}$-coordinated $\pi$-system of the indenyl framework. The dihedral angle between a plane defined by three carbon atoms of Ind (C1, C4 and C5) and a plane defined by atoms $\mathrm{C} 1$, $\mathrm{C} 10, \mathrm{O} 1$ and $\mathrm{O} 2$ is $3.8(2)^{\circ}$.

The crystal structure of $\mathbf{1 2}$ is stabilized by a sandwich $\pi-\pi$ stacking involving the unsubstituted indenyl ligands of two neighboring molecules. The distance between the centroid of the five-membered ring $[\mathrm{Cg}(\mathrm{C} 12-\mathrm{C} 16)]$ and a plane defined by the parallel five-membered ring $\left[\mathrm{Pl}\left(\mathrm{C} 12^{\prime}-\mathrm{C} 16^{\prime}\right)\right]$ is 3.3075(10) $\mathrm{A}$. A T-shaped interaction between the face of the six-membered ring of the substituted indenyl ligand (C2-C6) and the sixmembered ring vertex of the unsubstituted indenyl $\left(\mathrm{C} 17^{\prime}-\mathrm{H} 17^{\prime}\right)$ connects the molecules into zig-zag chains along the $b$-axis. The distance between the centroid $\mathrm{Cg}(\mathrm{C} 2-\mathrm{C} 6)$ and the carbon atom $\mathrm{C} 17^{\prime}$ is $3.532(2) \AA$.

Table 2 Geometric parameters of the molybdenum complexes ${ }^{a}$

\begin{tabular}{|c|c|c|c|c|c|}
\hline & 7 & 12 & 14 & 20 & $22 \cdot \mathrm{MeOH}$ \\
\hline $\mathrm{Mo}-\mathrm{Cg}\left(\mathrm{C}_{5}\right)$ & $2.0358(12)$ & $2.0203(10)$ & $2.0366(11)$ & $2.0229(10)$ & $2.0369(11)$ \\
\hline $\mathrm{Mo}-\mathrm{Cg}\left(\mathrm{C}_{3}\right)$ & $2.044(4)$ & $2.1423(19)$ & $2.053(3)$ & $2.047(3)$ & $2.049(3)$ \\
\hline $\mathrm{Mo}-\mathrm{C}(\mathrm{CO})$ & $1.936(3)$ & $1.943(2)$ & $1.933(3)$ & $1.931(2)$ & $1.939(3)$ \\
\hline & $1.950(3)$ & $1.952(2)$ & $1.934(3)$ & $1.954(2)$ & $1.943(3)$ \\
\hline $\mathrm{Cg}\left(\mathrm{C}_{5}\right)-\mathrm{Mo}-\mathrm{Cg}\left(\mathrm{C}_{3}\right)$ & $126.06(13)$ & $131.62(6)$ & $127.88(10)$ & $126.69(11)$ & $127.09(10)$ \\
\hline $\mathrm{C}(\mathrm{CO})-\mathrm{Mo}-\mathrm{C}(\mathrm{CO})$ & $80.26(12)$ & $79.22(9)$ & $79.15(13)$ & $82.79(9)$ & $78.04(11)$ \\
\hline$\Omega^{b}$ & $4.5(3)$ & $\begin{array}{l}4.4(2)^{d} \\
18.3(2)^{e}\end{array}$ & $3.4(3)$ & $4.5(2)$ & $3.7(3)$ \\
\hline$\Delta(\mathrm{M}-\mathrm{C})^{c}$ & $0.119(3)$ & $\begin{array}{l}0.114(2)^{d} \\
0.765(2)^{e}\end{array}$ & $0.106(3)$ & $0.104(2)$ & $0.105(3)$ \\
\hline
\end{tabular}

${ }^{a}$ Distances are given in $\AA$; angles and dihedral angles are given in ${ }^{\circ} .{ }^{b} \Omega$ is the envelope fold angle defined for the indenyl ligand as the angle between planes defined by $\mathrm{C} 1, \mathrm{C} 2$ and $\mathrm{C} 3$ and that of $\mathrm{C} 1, \mathrm{C} 3, \mathrm{C} 8$ and $\mathrm{C} 9 .^{22}{ }^{c} \Delta(\mathrm{M}-\mathrm{C})$ represents the differences in the metal-carbon bonds. It is defined for the indenyl compounds as the difference between the averages of the metal-carbon distances $\mathrm{M}-\mathrm{C} 8$ and $\mathrm{M}-\mathrm{C} 9$ and those of $\mathrm{M}-\mathrm{C} 1$, $\mathrm{M}-\mathrm{C} 2$, and $\mathrm{M}-\mathrm{C} 3{ }^{22}{ }^{d}$ Data for Ind. ${ }^{e}$ Data for $1-(\mathrm{MeOCO}) \mathrm{C}_{9} \mathrm{H}_{6}$. 


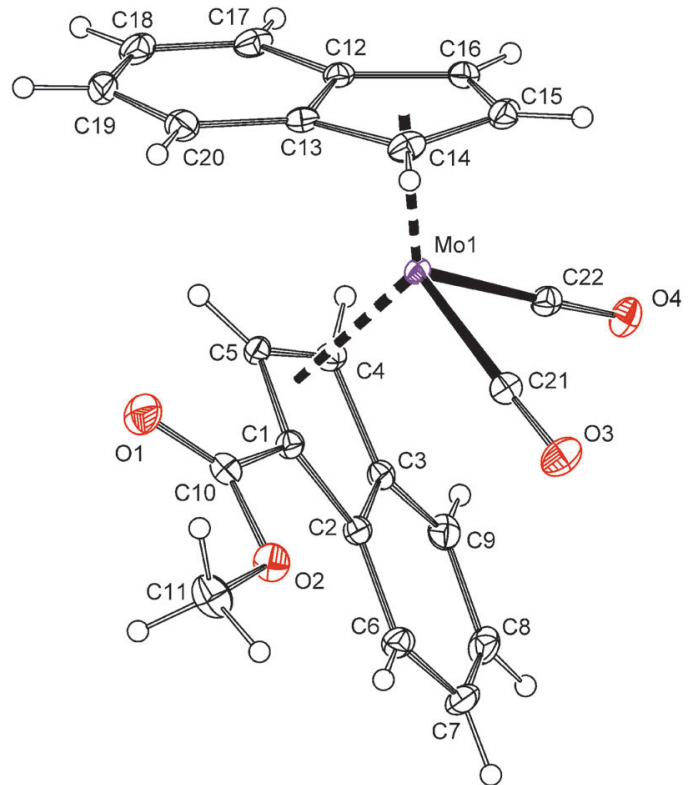

Fig. 2 ORTEP drawing of the molybdenum compound $\left[\left\{\eta^{3}-1-(\mathrm{MeO}-\right.\right.$ CO) $\left.\mathrm{C}_{9} \mathrm{H}_{6}\right\}\left(\eta^{5} \text {-Ind)Mo(CO) }\right)_{2}$ ] present in the crystal structure of 12. Thermal ellipsoids are drawn at the $30 \%$ probability level.

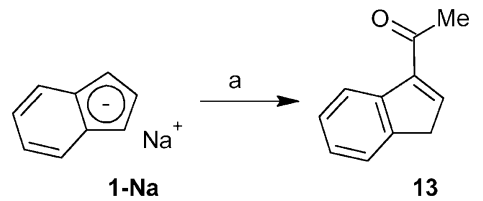

Scheme 5 Synthesis of acetyl-functionalized indene 13. Reagent: (a) MeCOOEt.

\section{Acetyl-functionalized indenyl molybdenum compound}

A similar strategy as described for indene $\mathbf{2}$ was utilized for the synthesis of the acetyl-functionalized derivative $3-(\mathrm{MeCO}) \mathrm{C}_{9} \mathrm{H}_{7}$ (13). Hence, the reaction of sodium indenide with ethyl acetate produced indene $\mathbf{1 3}$ in $38 \%$ isolated yield including a vacuum distillation step (Scheme 5). The protocol utilized inexpensive and readily available starting materials providing the product on a multigram scale. The vibrational spectra show a strong band of $\mathrm{CO}$ stretching in a range typical for the keto-group (IR: $1669 \mathrm{~cm}^{-1}$; Raman: $1666 \mathrm{~cm}^{-1}$ ). The ${ }^{1} \mathrm{H}$ and ${ }^{13} \mathrm{C}\left\{{ }^{1} \mathrm{H}\right\}$ NMR spectra reveal the appearance of an isomer with the acetyl function in the 3-position of the indene framework. This observation is in line with the X-ray analysis of a single crystal obtained by recrystallization from hexane at low temperature. Hence, the acetyl group is attached to the $\mathrm{sp}^{2}$ carbon atom of the indene framework, see Fig. 3. The bond length between the carbon atom $\mathrm{C} 1$ and the oxygen atom $\mathrm{O} 1$ is 1.222(2) $\AA$ that is within a range typical for the $\mathrm{CO}$ double bond.

Deprotonation of indene $\mathbf{1 3}$ with $n$-butyl lithium followed by addition of the allyl complex $\left[\left(\eta^{3}-\mathrm{C}_{3} \mathrm{H}_{5}\right) \mathrm{Mo}(\mathrm{CO})_{2}(\mathrm{NCMe})_{2} \mathrm{Cl}\right]$ (6) yields the desired $\eta^{5}$-indenyl complex $\left[\left(\eta^{3}-\mathrm{C}_{3} \mathrm{H}_{5}\right)\left\{\eta^{5}\right.\right.$-1-(MeCO) $\left.\mathrm{C}_{9} \mathrm{H}_{6}\right\}$ $\left.\mathrm{Mo}(\mathrm{CO})_{2}\right](\mathbf{1 4})$, see Scheme 6.

Infrared, Raman and ${ }^{1} \mathrm{H}$ NMR spectroscopy confirmed the successful assembly of the acetyl-functionalized indenyl

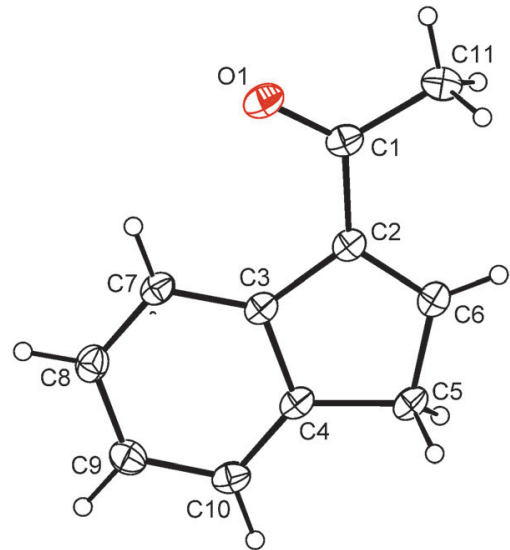

Fig. 3 ORTEP drawing of $3-(\mathrm{MeCO}) \mathrm{C}_{9} \mathrm{H}_{7}$ present in the crystal structure of 13 . Thermal ellipsoids are drawn at the $30 \%$ probability level.

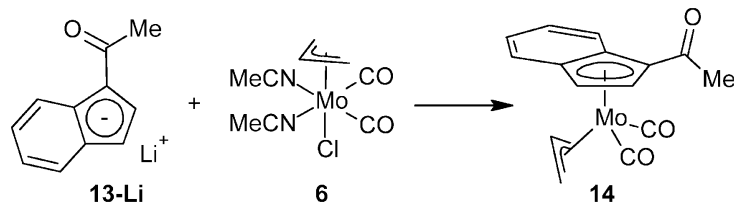

Scheme 6 Synthesis of an acetyl-functionalized indenyl molybdenum complex.

molybdenum framework. Hence, the vibrational spectra of compound 14 show, in addition to stretching bands of the carbonyl ligands, a characteristic band of the $\mathrm{C}=\mathrm{O}$ stretching at similar frequencies as observed for indene precursor 13, see Table 3. The ${ }^{1} \mathrm{H}$ NMR spectrum of compound $\mathbf{1 4}$ features a similar pattern as observed for the ester derivatives. The molar ratio of exo- and endo-conformers $(3: 1)$ is virtually the same as observed for the ester derivatives. This observation indicates that the abundance of given conformers is directed by the bulkiness of the modified indenyl ligand while electronic properties of the substituents play only a minor role.

$\mathrm{X}$-ray crystallographic structure determination confirmed that the acetyl-substituted molybdenum compound $\mathbf{1 4}$ is isostructural with ester-derivative 7 . The acetyl function is coplanar

Table 3 Summary of the infrared and Raman data for the acyl-and amide-functionalized molybdenum complexes ${ }^{a}$

\begin{tabular}{llllll}
\hline & Infrared & & & \multicolumn{2}{l}{ Raman } \\
\cline { 2 - 3 } \cline { 5 - 6 } & $\nu(\mathrm{C} \equiv \mathrm{O})$ & $\nu(\mathrm{C}=\mathrm{O})$ & & $\nu(\mathrm{C} \equiv \mathrm{O})$ & $\nu(\mathrm{C}=\mathrm{O})$ \\
\hline $\mathbf{1 4}$ & 1936 & 1660 & 1932 & 1666 \\
& 1858 & & 1836 & \\
20 & 1932 & 1635 & 1931 & 1637 \\
& 1848 & & 1850 & \\
21 & 1932 & 1633 & 1933 & 1635 \\
& 1859 & & 1866 & \\
22 & 1940 & 1667 & 1948 & 1664
\end{tabular}

${ }^{a}$ The stretching frequencies are given in $\mathrm{cm}^{-1}$. 


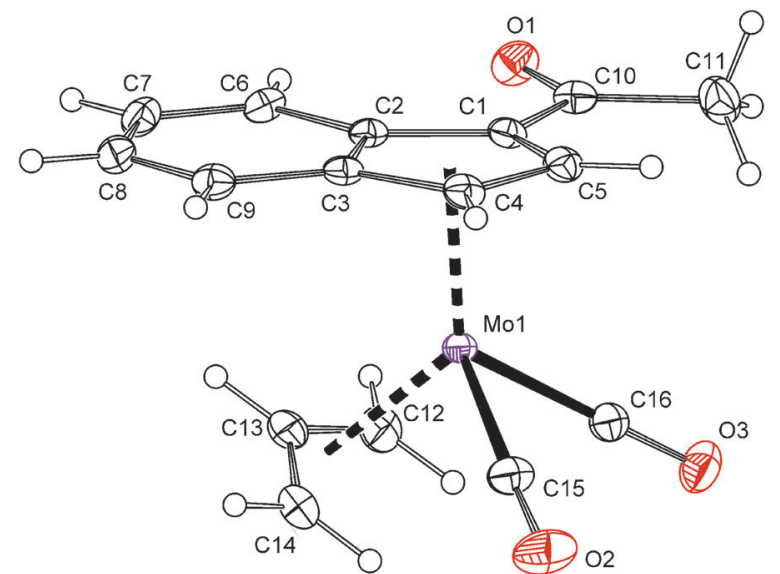

Fig. 4 ORTEP drawing of the molybdenum compound $\left[\left(\eta^{3}-\mathrm{C}_{3} \mathrm{H}_{5}\right)\left\{\eta^{5}-1-\right.\right.$ $\left.(\mathrm{MeCO}) \mathrm{C}_{9} \mathrm{H}_{6}\right\} \mathrm{Mo}(\mathrm{CO})_{2}$ ] present in the crystal structure of 14 . Thermal ellipsoids are drawn at the $30 \%$ probability level.

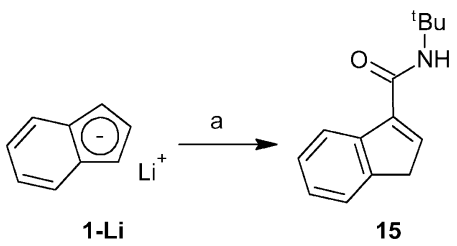

Scheme 7 Synthesis of carboxamide-functionalized indene 15. Reagent: (a) ${ }^{t}$ BuNCO.
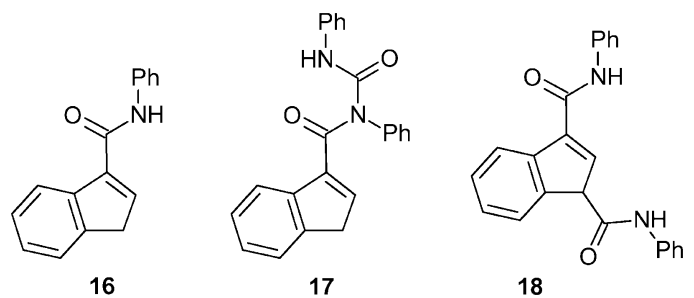

Scheme 8 Product of the reaction between 1-Li and PhNCO.

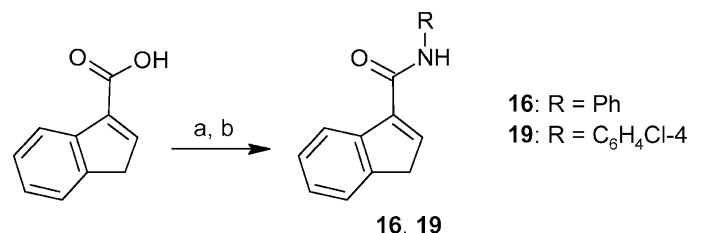

Scheme 9 Synthesis of carboxamide-functionalized indenes 16 and 19 Reagents: (a) $\mathrm{SOCl}_{2}$, (b) RNCO. with the indenyl framework. The dihedral angle between the C5 ring of indenyl and a plane defined by atoms C1, C10, O1 and $\mathrm{O} 2$ is $2.15(17)^{\circ}$ (Fig. 4).

\section{Amide-functionalized indenyl molybdenum compounds}

Finally, we have also investigated the scope of our synthetic protocol for compounds with amide-functions. The starting amide-substituted indenes were synthesized using two strategies. tert-Butyl derivative $\mathbf{1 5}$ was prepared by a reaction of lithium indenide (1-Li) with tert-butyl isocyanate, see Scheme 7. Rather inexpensive starting materials and an acceptable isolated yield (65\%) predestinate this procedure for the use on a multigram scale.

Unfortunately, the higher reactivity of aryl isocyanates precludes their use for the synthesis of aryl amides such as $\mathbf{1 6}$ and 19 (for structures, see Scheme 9). In fact, the reaction of phenyl isocyanate gives the acyl-substituted $N, N$-diphenyl urea 17 and the 1,3-disubstituted indene $\mathbf{1 8}$ as major outcomes in about $22 \%$ and $8 \%$ yields, respectively (based on indene). The desired 3-( $\mathrm{PhNHCO}) \mathrm{C}_{9} \mathrm{H}_{7}$ (16) appears only as a minor product (in $\sim 4 \%$ yield) that was separated from the reaction mixture by column chromatography (Scheme 8).

This led us to use $3-(\mathrm{ClCO}) \mathrm{C}_{9} \mathrm{H}_{7}$ prepared in situ for the synthesis of aryl amides $\mathbf{1 6}$ and 19, see Scheme 9. The carboxylic acid, 3-(HOCO) $\mathrm{C}_{9} \mathrm{H}_{7}$, was treated with thionyl chloride and the product reacted with anilines to give carboxamides 16 and 19 in satisfactory isolated yields.

Indenyl molybdenum compounds bearing the amide function group are accessible using the aforementioned protocol successfully used for the ester-derivatives. Deprotonation with $n$-butyl lithium followed by a metathesis reaction gave indenyl molybdenum compounds 20-22 in moderate isolated yields, see Scheme 10. The compounds were characterized by infrared, Raman and ${ }^{1} \mathrm{H}$ NMR spectroscopy. The structures of compounds 20 and 22 were determined by X-ray diffraction analysis. The vibration spectra of compounds 20-22 show stretching bands of the carbonyl ligands at frequencies similar to those observed for esterderivatives 7-10 ( $c f$. data in Tables 1 and 3). Frequencies of $\mathrm{N}-\mathrm{H}$ and $\mathrm{C}=\mathrm{O}$ stretching bands of the amide functions are also consistent with the desired $\eta^{5}$-coordination mode of the substituted indenyl ligands.

In case of compounds 20 and 22, a successful assembly of the $\left[\left(\eta^{3}-\mathrm{C}_{3} \mathrm{H}_{5}\right)\left(\eta^{5} \text {-Ind)Mo(CO) }\right)_{2}\right]$ moiety was further confirmed by the X-ray diffraction analysis, see Fig. 5 and 6 . The carboxamide-functionalized indenyl ligands are $\eta^{5}$ coordinated to the molybdenum as evidenced by ring slip parameters (Table 2). Surprisingly, only a very weak hydrogen bond $\left(\mathrm{N} 1-\mathrm{H} 1 \cdots \mathrm{O} 1^{\prime}\right)$ was observed in the crystal lattice of

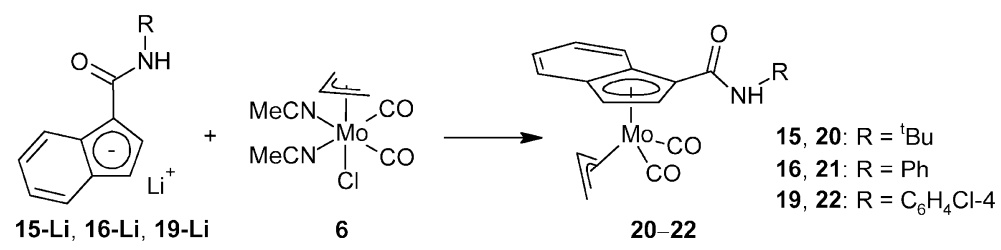

Scheme 10 Synthesis of amide-functionalized indenyl molybdenum complexes. 


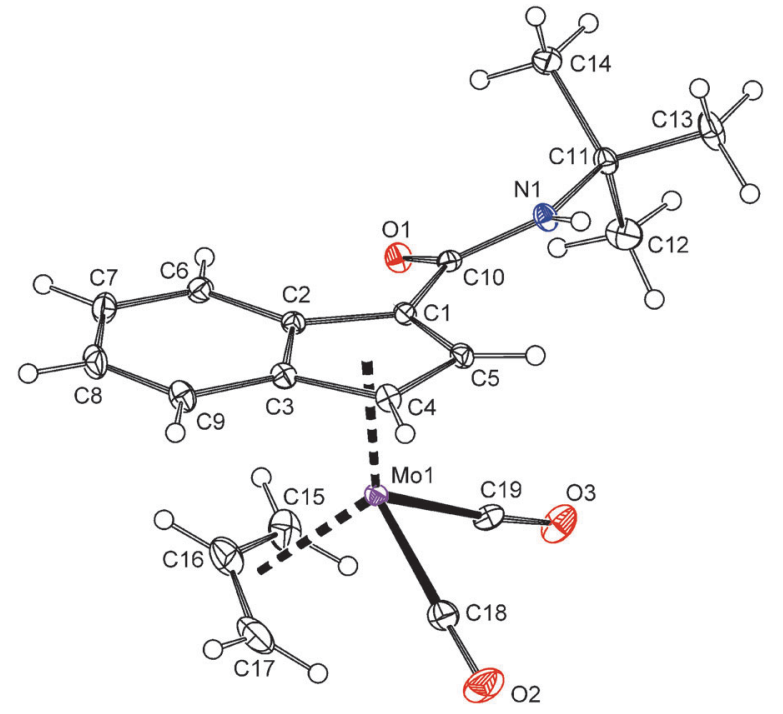

Fig. 5 ORTEP drawing of the molybdenum compound $\left[\left(\eta^{3}-\mathrm{C}_{3} \mathrm{H}_{5}\right)\left\{\eta^{5}-1-\right.\right.$ $\left({ }^{t} \mathrm{BuNHCO}\right) \mathrm{C}_{9} \mathrm{H}_{6} 3 \mathrm{Mo}(\mathrm{CO})_{2}$ ] present in the crystal structure of 20 . Thermal ellipsoids are drawn at the $30 \%$ probability level.

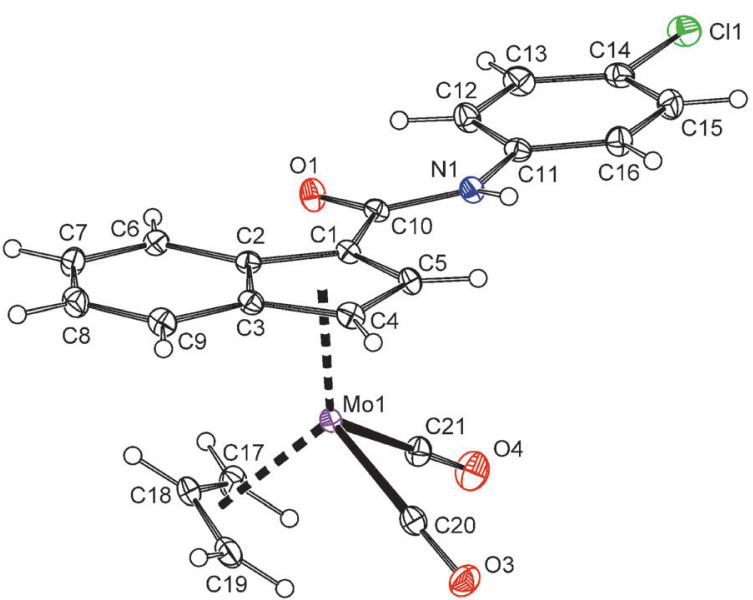

Fig. 6 ORTEP drawing of the molybdenum compound $\left[\left(\eta^{3}-\mathrm{C}_{3} \mathrm{H}_{5}\right)\left\{\eta^{5}-1-\right.\right.$ $\left(4-\mathrm{ClC}_{6} \mathrm{H}_{4} \mathrm{NHCO} \mathrm{C}_{9} \mathrm{H}_{6}\right\} \mathrm{Mo}(\mathrm{CO})_{2}$ ] present in the crystal structure of 22. $\mathrm{MeOH}$. Thermal ellipsoids are drawn at the $30 \%$ probability level.

compound 20 probably as a result of the steric hindrance of the bulky $t$-butyl group. The amide functions of neighboring molecules are connected into zig-zag chains along the $c$-axis. The distance between the nitrogen atom (N1) and the oxygen atom $\left(\mathrm{O}^{\prime}\right)$ of the neighboring amide function is 3.295(2) $\AA$. In case of $22 \cdot \mathrm{MeOH}$, the amide functions and the hydroxyl group of methanol are connected into chains along the $a$-axis by considerably stronger hydrogen bonds $\mathrm{N} 1-\mathrm{H} 1 \cdots \mathrm{O} 2$ and $\mathrm{O} 2-\mathrm{H} 2 \cdots \mathrm{O} 1^{\prime}$. The distances $\mathrm{N} 1 \cdots \mathrm{O} 2$ and $\mathrm{O}_{2} \cdots \mathrm{O}^{\prime}{ }^{\prime}$ are 2.936(3) and 2.714(3) ^, respectively.

\section{Conclusions}

The present study established a new family of ring-substituted indenyl complexes with an unprecedented substitution pattern.
The derivatives of $\left[\left(\eta^{3}-\mathrm{C}_{3} \mathrm{H}_{5}\right)\left(\eta^{5}\right.\right.$-Ind $\left.) \mathrm{Mo}(\mathrm{CO})_{2}\right]$ bearing a strong electron-withdrawing functional group attached to the indenyl framework were prepared by a convenient route starting from functionalized lithium indenides. Since the availability of the ligand precursor is often a limiting factor of a synthetic protocol and consequent applications, attention was further given to low-cost pathways giving the ester-, acyl- and amidefunctionalized indenes without the necessity of a tedious chromatographic purification step. On that count, one representative from each group was synthesized on the 6-14 g scale starting from common and inexpensive starting materials.

The herewith established substitution of the indenyl ligands with polar functional groups opens a novel pathway for chemical modifications of transition metal complexes and may accelerate their use in organic synthesis or catalysis.

\section{Experimental section}

\section{Methods and materials}

All operations were performed under nitrogen using conventional Schlenk-line techniques. The solvents were purified and dried by standard methods. ${ }^{25}$ The starting materials were available commercially or prepared according to literature procedures: $3-(\mathrm{HOCO}) \mathrm{C}_{9} \mathrm{H}_{7},{ }^{21}\left[\left(\eta^{3}-\mathrm{C}_{3} \mathrm{H}_{5}\right) \mathrm{Mo}(\mathrm{CO})_{2}(\mathrm{NCMe})_{2} \mathrm{Cl}\right]$ $(\mathbf{6}){ }^{18}\left[\left\{\left(\eta^{5}-\text { Ind }\right) \mathrm{Mo}(\mathrm{CO}){ }_{2}(\mu-\mathrm{Cl})\right\}_{2}\right](\mathbf{1 1}){ }^{20}$

\section{Measurements}

Infrared and Raman spectra were measured on a Nicolet iS50 FTIR spectrometer equipped with a Raman module. The infrared spectra were recorded in the $4000-400 \mathrm{~cm}^{-1}$ region (resolution $1 \mathrm{~cm}^{-1}$ ) using a Diamond Smart Orbit ATR. Raman spectra were recorded in the $4000-100 \mathrm{~cm}^{-1}$ region (resolution $2 \mathrm{~cm}^{-1}$ ) in glass capillaries. The excitation source consisted of a Nd:YAG laser emitting at $1064 \mathrm{~cm}^{-1} \cdot{ }^{1} \mathrm{H}$ and ${ }^{13} \mathrm{C}\left\{{ }^{1} \mathrm{H}\right\}$ NMR spectra were measured on a Bruker Avance 400 and a Bruker Avance 500 spectrometers, respectively, at room temperature. The chemical shifts are given in ppm relative to TMS.

\section{Synthesis of 3-(MeOCO) $\mathrm{C}_{9} \mathrm{H}_{7}$ (2)}

Sodium indenide (1-Na), freshly prepared from indene $(\mathbf{1} ; 11.7 \mathrm{~mL}$, $0.10 \mathrm{~mol})$ and sodium hydride $(2.4 \mathrm{~g}, 0.10 \mathrm{mmol})$ in THF $(150 \mathrm{~mL})$, was treated with dimethyl carbonate $(8.4 \mathrm{~mL}, 0.10 \mathrm{~mol})$ and heated under reflux overnight. After cooling to room temperature, the reaction mixture was poured on an ice/water mixture and extracted with diethyl ether. The combined organic phases were washed with water, dried with magnesium sulfate and volatiles were vacuum evaporated on a rotavapor. The crude product was vacuum distilled $\left(120^{\circ} \mathrm{C}, 660 \mathrm{~Pa}\right)$. Yield: $7.2 \mathrm{~g}(41 \mathrm{mmol}, 41 \%)$. Pale yellow oil. Anal. Calc. for $\mathrm{C}_{11} \mathrm{H}_{10} \mathrm{O}_{2}$ : C: 75.84; H: 5.79. Found: C: $75.72 ; \mathrm{H}: 5.85 .{ }^{1} \mathrm{H}$ NMR $\left(\mathrm{CDCl}_{3} ; 400 \mathrm{MHz} ; \delta \mathrm{ppm}\right)$ : $7.94\left(\mathrm{~d},{ }^{3} J\left({ }^{1} \mathrm{H},{ }^{1} \mathrm{H}\right)=7.7 \mathrm{~Hz}, 1 \mathrm{H}, \mathrm{C}_{9} H_{7}\right), 7.28\left(\mathrm{~d},{ }^{3} J\left({ }^{1} \mathrm{H},{ }^{1} \mathrm{H}\right)=7.5 \mathrm{~Hz}\right.$, $\left.1 \mathrm{H}, \mathrm{C}_{9} H_{7}\right), 7.24\left(\mathrm{~s}, 1 \mathrm{H}, \mathrm{C}_{9} H_{7}\right), 7.21\left(\mathrm{t},{ }^{3} J\left({ }^{1} \mathrm{H},{ }^{1} \mathrm{H}\right)=7.5 \mathrm{~Hz}, 1 \mathrm{H}\right.$, $\left.\mathrm{C}_{9} H_{7}\right), 7.10\left(\mathrm{t},{ }^{3} J\left({ }^{1} \mathrm{H},{ }^{1} \mathrm{H}\right)=7.5 \mathrm{~Hz}, 1 \mathrm{H}, \mathrm{C}_{9} H_{7}\right), 3.73(\mathrm{~s}, 3 \mathrm{H}$, $\left.\mathrm{COOCH}_{3}\right), 3.26\left(\mathrm{~s}, 2 \mathrm{H}, \mathrm{C}_{9} H_{7}\right) \cdot{ }^{13} \mathrm{C}\left\{{ }^{1} \mathrm{H}\right\}$ NMR $\left(\mathrm{CDCl}_{3} ; 101 \mathrm{MHz}\right.$; $\delta \mathrm{ppm}): 164.3\left(1 \mathrm{C}, \mathrm{C}_{\mathrm{q}}, C \mathrm{COMe}\right), 144.4\left(1 \mathrm{C}, \mathrm{CH}, C_{9} \mathrm{H}_{7}\right), 143.3$, 
140.6, $135.8\left(3 \times 1 \mathrm{C}, \mathrm{C}_{\mathrm{q}}, C_{9} \mathrm{H}_{7}\right), 126.5,125.4,123.6,122.3$ $\left(4 \times 1 \mathrm{C}, \mathrm{CH}, C_{9} \mathrm{H}_{7}\right), 51.3\left(1 \mathrm{C}, \mathrm{CH}_{3}, \mathrm{COOCH}_{3}\right), 38.2\left(1 \mathrm{C}, \mathrm{CH}_{2}\right.$, $\left.C_{9} \mathrm{H}_{7}\right)$. IR $\left(\mathrm{ATR}, \mathrm{cm}^{-1}\right): 1713 \mathrm{~s}\left[\nu(\mathrm{CO})_{\mathrm{C}=\mathrm{O}}\right]$. Raman (capillary, $\left.\mathrm{cm}^{-1}\right): 1715(4)\left[\nu(\mathrm{CO})_{\mathrm{C}=\mathrm{O}}\right]$.

\section{Reaction of 1-Na with ClCOOMe}

Sodium indenide (1-Na), freshly prepared from indene $(\mathbf{1}, \mathbf{1 1 . 7} \mathrm{mL}$, $0.10 \mathrm{~mol})$ and sodium hydride $(2.4 \mathrm{~g}, 0.10 \mathrm{~mol})$ in THF $(150 \mathrm{~mL})$, was treated with methyl chloroformate $(7.7 \mathrm{~mL}, 0.1 \mathrm{~mol})$ and stirred at room temperature overnight. The reaction mixture was poured on an ice/water mixture and extracted with diethyl ether. The combined organic phases were dried with magnesium sulfate and volatiles were evaporated on a rotavapor. Fraction distillation of the crude product gave 2 as a minor product (yield: $0.4 \mathrm{~g}$, $2.3 \mathrm{mmol}, 2 \%$ ) and $2 \mathrm{a}$ as the major product (yield: $3.5 \mathrm{~g}, 15 \mathrm{mmol}$, 15\%). 1,1-(MeOCO) ${ }_{2} \mathrm{C}_{9} \mathrm{H}_{6}$ (2a): colorless crystals. $\mathrm{Bp}=95{ }^{\circ} \mathrm{C}(10 \mathrm{~Pa})$. $\mathrm{Mp}=84{ }^{\circ} \mathrm{C}$. Anal. Calc. for $\mathrm{C}_{13} \mathrm{H}_{12} \mathrm{O}_{4}: \mathrm{C}: 67.23 ; \mathrm{H}: 5.21$. Found: C: 67.38; H: 5.14. ${ }^{1} \mathrm{H}$ NMR $\left(\mathrm{CDCl}_{3} ; 400 \mathrm{MHz} ; \delta \mathrm{ppm}\right): 7.69$ $\left(\mathrm{d},{ }^{3} J\left({ }^{1} \mathrm{H},{ }^{1} \mathrm{H}\right)=7.4 \mathrm{~Hz}, 1 \mathrm{H}, \mathrm{C}_{9} H_{6}\right), 7.36-7.24\left(\mathrm{~m}, 3 \mathrm{H}, \mathrm{C}_{9} H_{6}\right), 6.91$ $\left(\mathrm{d},{ }^{3} J\left({ }^{1} \mathrm{H},{ }^{1} \mathrm{H}\right)=5.5 \mathrm{~Hz}, 1 \mathrm{H}, \mathrm{C}_{9} H_{6}\right), 6.56\left(\mathrm{~d},{ }^{3} J\left({ }^{1} \mathrm{H},{ }^{1} \mathrm{H}\right)=5.5 \mathrm{~Hz}, 1 \mathrm{H}\right.$, $\left.\mathrm{C}_{9} H_{6}\right), 3.73$ (s, 6H, COOCH$\left.{ }_{3}\right) .{ }^{13} \mathrm{C}\left\{{ }^{1} \mathrm{H}\right\} \mathrm{NMR}\left(\mathrm{CDCl}_{3} ; 101 \mathrm{MHz}\right.$; $\delta$ ppm): 168.5 (2C, $\left.\mathrm{C}_{\mathrm{q}}, C O O M e\right), 143.8,139.9\left(2 \times 1 \mathrm{C}, \mathrm{C}_{\mathrm{q}}, C_{9} \mathrm{H}_{6}\right)$, 135.1, 133.5, 129.4 126.6, 125.6, $121.9\left(6 \times 1 \mathrm{C}, \mathrm{CH}, C_{9} \mathrm{H}_{6}\right), 53.5$ $\left(2 \mathrm{C}, \mathrm{CH}_{3}, \mathrm{COOCH}_{3}\right)$. IR (ATR, $\left.\mathrm{cm}^{-1}\right): 1743 \mathrm{~s}\left[\nu_{\mathrm{a}}(\mathrm{CO})_{\mathrm{C}=\mathrm{O}}\right], 1724 \mathrm{~s}$ $\left[\nu(\mathrm{CO})_{\mathrm{C}=\mathrm{O}}\right]$. Raman (capillary, $\left.\mathrm{cm}^{-1}\right): 1740(2) \quad\left[\nu_{\mathrm{a}}(\mathrm{CO})_{\mathrm{C}=\mathrm{O}}\right]$, $1724(2)\left[\nu_{\mathrm{s}}(\mathrm{CO})_{\mathrm{C}=\mathrm{O}}\right]$.

\section{Synthesis of 3-( $\left.\mathrm{MeOCH}_{2} \mathrm{CH}_{2} \mathrm{OCO}\right) \mathrm{C}_{9} \mathrm{H}_{7}$ (3)}

3 -(HOCO) $\mathrm{C}_{9} \mathrm{H}_{7}(0.80 \mathrm{~g} ; 5.0 \mathrm{mmol})$ was dissolved in the excess of thionyl chloride $(5 \mathrm{~mL})$ and stirred at room temperature for $1 \mathrm{~h}$ and then heated at $60{ }^{\circ} \mathrm{C}$ for $10 \mathrm{~min}$. The volatiles were vacuum evaporated. The sticky solid was treated with the excess of $\mathrm{MeOCH}_{2} \mathrm{CH}_{2} \mathrm{OH}(3.8 \mathrm{~g}, 50 \mathrm{mmol})$ and stirred for $2 \mathrm{~h}$. The reaction mixture was diluted with diethyl ether and the reaction was quenched with addition of aqueous solution sodium carbonate. The organic phase was separated and the water phase was washed with an additional portion of diethyl ether. The combined organic phases were washed with water, dried with magnesium sulfate. The volatiles were vacuum evaporated on a rotavapor and the crude product was purified by column chromatography on silica (diethyl ether/hexane = 1 : 9). Yield: $0.84 \mathrm{~g}$ (3.8 mmol, 77\%). Pale yellow oil. Anal. Calc. for $\mathrm{C}_{13} \mathrm{H}_{14} \mathrm{O}_{3}$ : C: 71.54; $\mathrm{H}$ : 6.47. Found: C: 71.59; $\mathrm{H}: 6.45$. ${ }^{1} \mathrm{H}$ NMR $\left(\mathrm{CDCl}_{3} ; 400 \mathrm{MHz} ; \delta \mathrm{ppm}\right): 8.04\left(\mathrm{~d},{ }^{3} J\left({ }^{1} \mathrm{H},{ }^{1} \mathrm{H}\right)=7.7\right.$ $\left.\mathrm{Hz}, 1 \mathrm{H}, \mathrm{C}_{9} H_{7}\right), 7.49\left(\mathrm{t},{ }^{3} J\left({ }^{1} \mathrm{H},{ }^{1} \mathrm{H}\right)=2.0 \mathrm{~Hz}, 1 \mathrm{H}, \mathrm{C}_{9} H_{7}\right), 7.46$ $\left(\mathrm{d},{ }^{3} J\left({ }^{1} \mathrm{H},{ }^{1} \mathrm{H}\right)=7.5 \mathrm{~Hz}, 1 \mathrm{H}, \mathrm{C}_{9} H_{7}\right), 7.34\left(\mathrm{t},{ }^{3} J\left({ }^{1} \mathrm{H},{ }^{1} \mathrm{H}\right)=7.5 \mathrm{~Hz}\right.$, $\left.1 \mathrm{H}, \mathrm{C}_{9} H_{7}\right), 7.25\left(\mathrm{td},{ }^{3} J\left({ }^{1} \mathrm{H},{ }^{1} \mathrm{H}\right)=7.5 \mathrm{~Hz},{ }^{4} J\left({ }^{1} \mathrm{H},{ }^{1} \mathrm{H}\right)=1.2 \mathrm{~Hz}, 1 \mathrm{H}\right.$, $\left.\mathrm{C}_{9} H_{7}\right), 4.45\left(\mathrm{t},{ }^{3} J\left({ }^{1} \mathrm{H},{ }^{1} \mathrm{H}\right)=4.7 \mathrm{~Hz}, 2 \mathrm{H}, \mathrm{CH}_{2}\right), 3.72\left(\mathrm{t},{ }^{3} J\left({ }^{1} \mathrm{H},{ }^{1} \mathrm{H}\right)=\right.$ $\left.4.7 \mathrm{~Hz}, 2 \mathrm{H}, \mathrm{CH}_{2}\right), 3.51\left(\mathrm{~d},{ }^{3} J\left({ }^{1} \mathrm{H},{ }^{1} \mathrm{H}\right)=2.0 \mathrm{~Hz}, 2 \mathrm{H}, \mathrm{C}_{9} H_{7}\right), 3.42$ (s, 3H, $\left.\mathrm{OCH}_{3}\right) .{ }^{13} \mathrm{C}\left\{{ }^{1} \mathrm{H}\right\} \mathrm{NMR}\left(\mathrm{CDCl}_{3} ; 101 \mathrm{MHz} ; \delta \mathrm{ppm}\right): 164.2$ (1C, $\left.C_{\mathrm{q}}, C O O M e\right), 145.0$ (1C, CH, $\left.C_{9} \mathrm{H}_{7}\right), 143.5,140.9,136.2$ $\left(3 \times 1 \mathrm{C}, \mathrm{C}_{\mathrm{q}}, C_{9} \mathrm{H}_{7}\right), 126.9,125.7,124.0,122.7(4 \times 1 \mathrm{C}, \mathrm{CH}$, $\left.C_{9} \mathrm{H}_{7}\right), 70.8,63.7\left(2 \times 1 \mathrm{C}, \mathrm{CH}_{2}, \mathrm{CH}_{2}\right), 59.2\left(1 \mathrm{C}, \mathrm{CH}_{3}, \mathrm{OCH}_{3}\right), 38.6$ $\left(1 \mathrm{C}, \mathrm{CH}_{2}, C_{9} \mathrm{H}_{7}\right)$. IR (ATR, $\left.\mathrm{cm}^{-1}\right): 1713 \mathrm{~s}\left[\nu(\mathrm{CO})_{\mathrm{C}=\mathrm{O}}\right]$. Raman (capillary, $\left.\mathrm{cm}^{-1}\right)$ : $1717(3)\left[\nu(\mathrm{CO})_{\mathrm{C}=\mathrm{O}}\right]$.

\section{Synthesis of 3-( $\left.\mathrm{PhCH}_{2} \mathrm{OCO}\right) \mathrm{C}_{9} \mathrm{H}_{7}$ (4)}

The steps of synthesis followed the procedure for compound 3. Reagents: 3 -( $\mathrm{HOCO}) \mathrm{C}_{9} \mathrm{H}_{7}(0.80 \mathrm{~g}, 5.0 \mathrm{mmol}), \mathrm{PhCH}_{2} \mathrm{OH}$ (5.4 g, $50 \mathrm{mmol}$ ). The crude product was purified by column chromatography on silica (diethyl ether/hexane $=1: 9$ ). Yield: $1.0 \mathrm{~g}$ (4.0 mmol, 80\%). Pale yellow oil. Anal. Calc. for $\mathrm{C}_{17} \mathrm{H}_{14} \mathrm{O}_{2}$ : C: 81.58; H: 5.64. Found: $\mathrm{C}: 81.51 ; \mathrm{H}: 5.80 .{ }^{1} \mathrm{H} \mathrm{NMR}\left(\mathrm{CDCl}_{3}\right.$; $400 \mathrm{MHz} ; \delta$ ppm): $8.06\left(\mathrm{~d},{ }^{3} J\left({ }^{1} \mathrm{H},{ }^{1} \mathrm{H}\right)=7.7 \mathrm{~Hz}, 1 \mathrm{H}, \mathrm{C}_{9} H_{7}\right), 7.69$ $\left(\mathrm{t},{ }^{3} J\left({ }^{1} \mathrm{H},{ }^{1} \mathrm{H}\right)=1.8 \mathrm{~Hz}, 1 \mathrm{H}, \mathrm{C}_{9} H_{7}\right), 7.49-7.32\left(\mathrm{~m}, 2 \mathrm{H}\right.$ of $\mathrm{C}_{9} H_{7}$ and $5 \mathrm{H}$ of $\left.\mathrm{C}_{6} H_{5}\right), 7.26\left(\mathrm{t},{ }^{3} J\left({ }^{1} \mathrm{H},{ }^{1} \mathrm{H}\right)=7.4 \mathrm{~Hz}, 1 \mathrm{H}, \mathrm{C}_{9} H_{7}\right), 5.37(\mathrm{~s}, 2 \mathrm{H}$, $\left.\mathrm{CH}_{2} \mathrm{Ph}\right), 3.52\left(\mathrm{~d},{ }^{3} J\left({ }^{1} \mathrm{H},{ }^{1} \mathrm{H}\right)=1.8 \mathrm{~Hz}, 2 \mathrm{H}, \mathrm{C}_{9} H_{7}\right) .{ }^{13} \mathrm{C}\left\{{ }^{1} \mathrm{H}\right\} \mathrm{NMR}$ $\left(\mathrm{CDCl}_{3} ; 101 \mathrm{MHz} ; \delta \mathrm{ppm}\right): 164.1$ (1C, $\left.C_{\mathrm{q}}, C O O M e\right), 145.1$ $\left(1 \mathrm{C}, \mathrm{CH}, C_{9} \mathrm{H}_{7}\right), 143.5,140.9,136.3\left(3 \times 1 \mathrm{C}, \mathrm{C}_{\mathrm{q}}, C_{9} \mathrm{H}_{7}\right), 128.8$ $\left(2 \mathrm{C}, C_{6} \mathrm{H}_{5}\right), 128.4\left(1 \mathrm{C}, C_{6} \mathrm{H}_{5}\right), 128.3\left(2 \mathrm{C}, C_{6} \mathrm{H}_{5}\right), 126.9,125.8$, 124.0, $122.7\left(4 \times 1 \mathrm{C}, \mathrm{CH}, C_{9} \mathrm{H}_{7}\right), 66.4\left(1 \mathrm{C}, \mathrm{CH}_{2}, \mathrm{CH}_{2}\right), 38.6(1 \mathrm{C}$, $\left.\mathrm{CH}_{2}, C_{9} \mathrm{H}_{7}\right)$. IR $\left(\right.$ ATR, $\left.\mathrm{cm}^{-1}\right)$ : $1713 \mathrm{~s}\left[\nu(\mathrm{CO})_{\mathrm{C}=\mathrm{O}}\right]$. Raman (capillary, $\left.\mathrm{cm}^{-1}\right): 1717(2)\left[\nu(\mathrm{CO})_{\mathrm{C}=\mathrm{O}}\right]$.

\section{Synthesis of 3-(4- $\left.\mathrm{MeOC}_{6} \mathrm{H}_{4} \mathrm{OCO}\right) \mathrm{C}_{9} \mathrm{H}_{7}$ (5)}

The steps of synthesis followed the procedure for compound 3 . Reagents: $3-(\mathrm{HOCO}) \mathrm{C}_{9} \mathrm{H}_{7}(0.80 \mathrm{~g}, 5.0 \mathrm{mmol}), 4-\mathrm{MeOC}_{6} \mathrm{H}_{4} \mathrm{OH}$ (1.1 g, $9 \mathrm{mmol})$. The crude product was purified by column chromatography on silica (diethyl ether/hexane $=1: 9$ ). Yield: $0.72 \mathrm{~g}$ (2.7 mmol, 54\%). Colorless crystals. $\mathrm{Mp}=110{ }^{\circ} \mathrm{C}$. Anal. Calc. for $\mathrm{C}_{17} \mathrm{H}_{14} \mathrm{O}_{3}$ : C: 76.68; $\mathrm{H}: 5.30$. Found: C: 76.75; H: 5.33. ${ }^{1} \mathrm{H} \mathrm{NMR}\left(\mathrm{CDCl}_{3} ; 400 \mathrm{MHz} ; \delta \mathrm{ppm}\right): 8.08\left(\mathrm{~d},{ }^{3} J\left({ }^{1} \mathrm{H},{ }^{1} \mathrm{H}\right)=7.6 \mathrm{~Hz}\right.$, $\left.1 \mathrm{H}, \mathrm{C}_{9} H_{7}\right), 7.67\left(\mathrm{t},{ }^{3} J\left({ }^{1} \mathrm{H},{ }^{1} \mathrm{H}\right)=2.0 \mathrm{~Hz}, 1 \mathrm{H}, \mathrm{C}_{9} H_{7}\right), 7.50$ $\left(\mathrm{d},{ }^{3} J\left({ }^{1} \mathrm{H},{ }^{1} \mathrm{H}\right)=7.4 \mathrm{~Hz}, 1 \mathrm{H}, \mathrm{C}_{9} H_{7}\right), 7.36\left(\mathrm{t},{ }^{3} J\left({ }^{1} \mathrm{H},{ }^{1} \mathrm{H}\right)=7.6 \mathrm{~Hz}\right.$, $\left.1 \mathrm{H}, \mathrm{C}_{9} H_{7}\right), 7.28\left(\mathrm{td},{ }^{3} J\left({ }^{1} \mathrm{H},{ }^{1} \mathrm{H}\right)=7.5 \mathrm{~Hz},{ }^{4} J\left({ }^{1} \mathrm{H},{ }^{1} \mathrm{H}\right)=1.2 \mathrm{~Hz}, 1 \mathrm{H}\right.$, $\left.\mathrm{C}_{9} H_{7}\right), 7.13\left(\mathrm{~d},{ }^{3} J\left({ }^{1} \mathrm{H},{ }^{1} \mathrm{H}\right)=9.1 \mathrm{~Hz}, 2 \mathrm{H}, \mathrm{C}_{6} H_{4}\right), 7.93\left(\mathrm{~d},{ }^{3} J\left({ }^{1} \mathrm{H},{ }^{1} \mathrm{H}\right)=\right.$ $\left.9.1 \mathrm{~Hz}, 2 \mathrm{H}, \mathrm{C}_{6} H_{4}\right), 3.81\left(\mathrm{~s}, 3 \mathrm{H}, \mathrm{OCH}_{3}\right), 3.60\left(\mathrm{~d},{ }^{3} \mathrm{~J}\left({ }^{1} \mathrm{H},{ }^{1} \mathrm{H}\right)=2.0 \mathrm{~Hz}\right.$, $\left.2 \mathrm{H}, \mathrm{C}_{9} H_{7}\right) .{ }^{13} \mathrm{C}\left\{{ }^{1} \mathrm{H}\right\}$ NMR $\left(\mathrm{CDCl}_{3} ; 101 \mathrm{MHz} ; \delta \mathrm{ppm}\right): 162.9$ (1C, $\left.C_{\mathrm{q}}, C O O M \mathrm{e}\right), 157.6\left(1 \mathrm{C}, \mathrm{C}_{\mathrm{q}}, C_{6} \mathrm{H}_{4}\right), 146.3\left(1 \mathrm{C}, \mathrm{CH}, C_{9} \mathrm{H}_{7}\right), 144.3$ $\left(1 \mathrm{C}, \mathrm{C}_{\mathrm{q}}, C_{6} \mathrm{H}_{4}\right), 143.5,140.7,136.0\left(3 \times 1 \mathrm{C}, \mathrm{C}_{\mathrm{q}}, C_{9} \mathrm{H}_{7}\right), 127.0$, 126.0, 124.1, $122.7\left(4 \times 1 \mathrm{C}, \mathrm{CH}, C_{9} \mathrm{H}_{7}\right), 122.7,114.8(2 \times 2 \mathrm{C}, \mathrm{CH}$, $\left.C_{6} \mathrm{H}_{4}\right), 55.9\left(1 \mathrm{C}, \mathrm{CH}_{3}, \mathrm{OCH}_{3}\right), 38.9\left(1 \mathrm{C}, \mathrm{CH}_{2}, C_{9} \mathrm{H}_{7}\right)$. IR (ATR, $\left.\mathrm{cm}^{-1}\right): 1725 \mathrm{~s}\left[\nu(\mathrm{CO})_{\mathrm{C}=\mathrm{O}}\right]$. Raman (capillary, $\left.\mathrm{cm}^{-1}\right): 1727(8)$ $\left[\nu(\mathrm{CO})_{\mathrm{C}=\mathrm{O}}\right]$.

\section{Synthesis of $\left[\left(\eta^{3}-\mathrm{C}_{3} \mathrm{H}_{5}\right)\left\{\eta^{5}-1-(\mathrm{MeOCO}) \mathrm{C}_{9} \mathrm{H}_{6}\right\} \mathrm{Mo}(\mathrm{CO})_{2}\right]$ (7)}

3-(MeOCO) $\mathrm{C}_{9} \mathrm{H}_{7}(2 ; 0.87 \mathrm{~g}, 5 \mathrm{mmol})$ was dissolved with $30 \mathrm{~mL}$ of THF, cooled at $0{ }^{\circ} \mathrm{C}$ and treated dropwise with $3.1 \mathrm{~mL}$ of ${ }^{n} \mathrm{BuLi}$ $\left(1.6 \mathrm{~mol} \mathrm{~L}^{-1}\right)$. The reaction mixture was stirred for $1 \mathrm{~h}$ and then added dropwise to the THF solution of $\left[\left(\eta^{3}-\mathrm{C}_{3} \mathrm{H}_{5}\right) \mathrm{Mo}(\mathrm{CO})_{2}\right.$ $\left.(\mathrm{NCMe})_{2} \mathrm{Cl}\right](6 ; 1.55 \mathrm{~g}, 5 \mathrm{mmol})$ precooled to $-80{ }^{\circ} \mathrm{C}$. The reaction mixture was stirred at room temperature overnight and then vacuum evaporated to dryness. The solid residue was washed with cold hexane $(10 \mathrm{~mL})$ and extracted several times with hot hexane. The volatiles were vacuum evaporated and product was vacuum dried. Yield: $1.24 \mathrm{~g}$ (3.4 mmol, 68\%). Yellow powder. $\mathrm{Mp}=89{ }^{\circ} \mathrm{C}$. Anal. Calc. for $\mathrm{C}_{16} \mathrm{H}_{14} \mathrm{MoO}_{4}$ : C: 52.47; H: 3.85. Found: C: $52.29 ; \mathrm{H}: 3.80 .{ }^{1} \mathrm{H} \mathrm{NMR}\left(\mathrm{CDCl}_{3}, 400 \mathrm{MHz}, \delta \mathrm{ppm}\right.$; $3: 1$ mixture of $7 \mathbf{a}\left(\right.$ exo- $\left.\mathrm{C}_{3} \mathrm{H}_{5}\right)$ and $7 \mathbf{b}\left(\right.$ endo $\left.\left.-\mathrm{C}_{3} \mathrm{H}_{5}\right)\right)$ : 7.86-7.80 $\left(\mathrm{m}, 1 \mathrm{H}\right.$ of $\mathbf{a}$ and $1 \mathrm{H}$ of $\mathbf{b}, \mathrm{H}^{4,7}$ of $\left.\mathrm{C}_{9} H_{6}\right), 7.30-7.05(\mathrm{~m}, 3 \mathrm{H}$ of $\mathbf{a}$ and $3 \mathrm{H}$ of $\mathbf{b}, \mathrm{H}^{4-7}$ of $\mathrm{C}_{9} H_{6}$ ), 6.21 (s-br, $1 \mathrm{H}$ of $\mathbf{a}, \mathrm{H}^{2,3}$ of $\mathrm{C}_{9} H_{6}$ ), 6.11 
(s-br, $1 \mathrm{H}$ of b, $\mathrm{H}^{2,3}$ of $\mathrm{C}_{9} H_{6}$ ), 6.05 (s-br, $1 \mathrm{H}$ of $\mathbf{a}, \mathrm{H}^{2,3}$ of $\mathrm{C}_{9} H_{6}$ ), 5.98 (s-br, $1 \mathrm{H}$ of $\mathbf{b}, \mathrm{H}^{2,3}$ of $\mathrm{C}_{9} H_{6}$ ), $3.94\left(\mathrm{~s}, 3 \mathrm{H}\right.$ of $\mathbf{a}$ and $3 \mathrm{H}$ of $\mathbf{b}, \mathrm{CH}_{3}$ ), 3.50 (s-br, $1 \mathrm{H}$ of $\mathbf{b}, \mathrm{C}_{3} H_{5}$ ), 3.31 (s-br, $2 \mathrm{H}$ of $\mathbf{b}, \mathrm{C}_{3} H_{5}$ ), 2.33 $\left(\mathrm{d},{ }^{3} J\left({ }^{1} \mathrm{H},{ }^{1} \mathrm{H}\right)=6.0 \mathrm{~Hz}, 1 \mathrm{H}\right.$ of a, syn of $\left.\mathrm{C}_{3} H_{5}\right), 2.16\left(\mathrm{~d},{ }^{3} J\left({ }^{1} \mathrm{H},{ }^{1} \mathrm{H}\right)=\right.$ $6.0 \mathrm{~Hz}, 1 \mathrm{H}$ of a, syn of $\left.\mathrm{C}_{3} H_{5}\right), 1.03\left(\mathrm{~d},{ }^{3} J\left({ }^{1} \mathrm{H},{ }^{1} \mathrm{H}\right)=10.8 \mathrm{~Hz}, 1 \mathrm{H}\right.$ of a, anti of $\left.\mathrm{C}_{3} H_{5}\right), 0.83\left(\mathrm{~d},{ }^{3} J\left({ }^{1} \mathrm{H},{ }^{1} \mathrm{H}\right)=10.8 \mathrm{~Hz}, 1 \mathrm{H}\right.$ of a, anti of $\left.\mathrm{C}_{3} H_{5}\right)$, $0.72\left(\mathrm{~m}, 1 \mathrm{H}\right.$ of a, meso of $\left.\mathrm{C}_{3} H_{5}\right),-0.11\left(\mathrm{~d},{ }^{3} J\left({ }^{1} \mathrm{H},{ }^{1} \mathrm{H}\right)=8.4 \mathrm{~Hz}, 1 \mathrm{H}\right.$ of $\mathbf{b}$, anti of $\left.\mathrm{C}_{3} \mathrm{H}_{5}\right),-1.11\left(\mathrm{~d},{ }^{3} J\left({ }^{1} \mathrm{H},{ }^{1} \mathrm{H}\right)=8.4 \mathrm{~Hz}, 1 \mathrm{H}\right.$ of $\mathbf{b}$, anti of $\left.\mathrm{C}_{3} H_{5}\right)$. IR (ATR, $\left.\mathrm{cm}^{-1}\right): 1934 \mathrm{vs}\left[\nu_{\mathrm{a}}(\mathrm{CO})_{\mathrm{C} \equiv \mathrm{o}}\right], 1866 \mathrm{vs}\left[\nu_{\mathrm{s}}(\mathrm{CO})_{\mathrm{C} \equiv \mathrm{o}}\right]$, $1714 \mathrm{~s}\left[\nu(\mathrm{CO})_{\mathrm{C}=\mathrm{O}}\right]$. Raman (capillary, $\left.\mathrm{cm}^{-1}\right): 1944(3)\left[\nu_{\mathrm{a}}(\mathrm{CO})_{\mathrm{C} \equiv \mathrm{o}}\right]$, $1861(10)\left[\nu_{\mathrm{s}}(\mathrm{CO})_{\mathrm{C} \equiv \mathrm{o}}\right], 1715(9)\left[\nu(\mathrm{CO})_{\mathrm{C}=\mathrm{O}}\right]$. Single crystals suitable for X-ray diffraction analysis were obtained by recrystallization of the product from hexane.

\section{Synthesis of $\left[\left(\eta^{3}-\mathrm{C}_{3} \mathrm{H}_{5}\right)\left\{\eta^{5}-1-\left(\mathrm{MeOCH}_{2} \mathrm{CH}_{2} \mathrm{OCO}\right) \mathrm{C}_{9} \mathrm{H}_{6}\right\} \mathrm{Mo}(\mathrm{CO})_{2}\right](8)$}

The steps of synthesis followed the procedure for compound 7 . Reagents: 3-( $\left.\mathrm{MeOCH}_{2} \mathrm{CH}_{2} \mathrm{OCO}\right) \mathrm{C}_{9} \mathrm{H}_{7}(3 ; 0.44 \mathrm{~g}, 2 \mathrm{mmol}), 1.3 \mathrm{~mL}$ of ${ }^{n} \mathrm{BuLi}\left(1.6 \mathrm{~mol} \mathrm{~L}^{-1}\right),\left[\left(\eta^{3}-\mathrm{C}_{3} \mathrm{H}_{5}\right) \mathrm{Mo}(\mathrm{CO})_{2}(\mathrm{NCMe})_{2} \mathrm{Cl}\right](6 ; 0.62 \mathrm{~g}$, $2 \mathrm{mmol})$. Yield: $0.36 \mathrm{~g}$ (0.88 mmol, 44\%). Yellow viscous oil. Anal. Calc. for $\mathrm{C}_{18} \mathrm{H}_{18} \mathrm{MoO}_{5}$ : C: 52.69; H: 4.42. Found: C: 52.65; H: 4.48. ${ }^{1} \mathrm{H}$ NMR $\left(\mathrm{CDCl}_{3}, 400 \mathrm{MHz}, \delta\right.$ ppm; $3: 1$ mixture of 8a $\left(\right.$ exo $\left.-\mathrm{C}_{3} \mathrm{H}_{5}\right)$ and $\mathbf{8 b}\left(\right.$ endo $\left.\left.-\mathrm{C}_{3} \mathrm{H}_{5}\right)\right)$ : 7.86-7.78 (m, $1 \mathrm{H}$ of a and $1 \mathrm{H}$ of $\mathbf{b}, \mathrm{H}^{4,7}$ of $\left.\mathrm{C}_{9} \mathrm{H}_{6}\right), 7.40-7.00\left(\mathrm{~m}, 3 \mathrm{H}\right.$ of $\mathbf{a}$ and $3 \mathrm{H} \mathrm{of} \mathbf{b}, \mathrm{H}^{4-7}$ of $\mathrm{C}_{9} H_{6}$ ), 6.26 (s-br, $1 \mathrm{H}$ of $\mathbf{a}, \mathrm{H}^{2,3}$ of $\mathrm{C}_{9} H_{6}$ ), 6.16 (s-br, $1 \mathrm{H}$ of $\mathbf{b}, \mathrm{H}^{2,3}$ of $\mathrm{C}_{9} \mathrm{H}_{6}$ ), 6.05 (s-br, $1 \mathrm{H}$ of $\mathbf{a}, \mathrm{H}^{2,3}$ of $\mathrm{C}_{9} \mathrm{H}_{6}$ ), 5.98 (s-br, $1 \mathrm{H}$ of $\mathbf{b}$, $\mathrm{H}^{2,3}$ of $\left.\mathrm{C}_{9} \mathrm{H}_{6}\right), 4.51\left(\mathrm{~m}, 2 \mathrm{H}\right.$ of $\mathbf{a}$ and $2 \mathrm{H}$ of $\left.\mathbf{b}, \mathrm{CH}_{2}\right), 3.73(\mathrm{~m}, 2 \mathrm{H}$ of $\mathbf{a}$ and $2 \mathrm{H}$ of $\mathbf{b}, \mathrm{CH}_{2}$ ), 3.71 (s-br, $1 \mathrm{H}$ of $\left.\mathbf{b}, \mathrm{C}_{3} \mathrm{H}_{5}\right), 3.59$ (s-br, $2 \mathrm{H}$ of $\left.\mathbf{b}, \mathrm{C}_{3} \mathrm{H}_{5}\right), 3.43\left(\mathrm{~s}, 3 \mathrm{H}\right.$ of $\mathbf{a}$ and $3 \mathrm{H}$ of $\left.\mathbf{b}, \mathrm{CH}_{3}\right), 2.36\left(\mathrm{~d},{ }^{3} J\left({ }^{1} \mathrm{H},{ }^{1} \mathrm{H}\right)=\right.$ $5.7 \mathrm{~Hz}, 1 \mathrm{H}$ of a, syn of $\left.\mathrm{C}_{3} \mathrm{H}_{5}\right), 2.15\left(\mathrm{~d},{ }^{3} \mathrm{~J}\left({ }^{1} \mathrm{H},{ }^{1} \mathrm{H}\right)=5.7 \mathrm{~Hz}, 1 \mathrm{H}\right.$ of a, syn of $\left.\mathrm{C}_{3} H_{5}\right), 1.03\left(\mathrm{~d},{ }^{3} \mathrm{~J}\left({ }^{1} \mathrm{H},{ }^{1} \mathrm{H}\right)=10.8 \mathrm{~Hz}, 1 \mathrm{H}\right.$ of a, anti of $\left.\mathrm{C}_{3} H_{5}\right)$, $0.83\left(\mathrm{~d},{ }^{3} J\left({ }^{1} \mathrm{H},{ }^{1} \mathrm{H}\right)=10.8 \mathrm{~Hz}, 1 \mathrm{H}\right.$ of $\mathbf{a}$, anti of $\left.\mathrm{C}_{3} H_{5}\right), 0.75(\mathrm{~m}, 1 \mathrm{H}$ of a, meso of $\left.\mathrm{C}_{3} \mathrm{H}_{5}\right),-0.03\left(\mathrm{~d},{ }^{3} J\left({ }^{1} \mathrm{H},{ }^{1} \mathrm{H}\right)=8.8 \mathrm{~Hz}, 1 \mathrm{H}\right.$ of $\mathbf{b}$, anti of $\left.\mathrm{C}_{3} H_{5}\right),-1.17\left(\mathrm{~d},{ }^{3} J\left({ }^{1} \mathrm{H},{ }^{1} \mathrm{H}\right)=8.8 \mathrm{~Hz}, 1 \mathrm{H}\right.$ of $\mathbf{b}$, anti of $\left.\mathrm{C}_{3} H_{5}\right)$. IR $\left(\mathrm{ATR}, \mathrm{cm}^{-1}\right): 1947 \mathrm{vs}\left[\nu_{\mathrm{a}}(\mathrm{CO})_{\mathrm{C} \equiv \mathrm{o}}\right], 1869 \mathrm{vs}\left[\nu_{\mathrm{s}}(\mathrm{CO})_{\mathrm{C} \equiv \mathrm{o}}\right], 1712 \mathrm{~s}$ $\left[\nu(\mathrm{CO})_{\mathrm{C}=\mathrm{O}}\right]$.

\section{Synthesis of $\left[\left(\eta^{3}-\mathrm{C}_{3} \mathrm{H}_{5}\right)\left\{\eta^{5}-1-\left(\mathrm{PhCH}_{2} \mathrm{OCO}\right) \mathrm{C}_{9} \mathrm{H}_{6}\right\} \mathrm{Mo}(\mathrm{CO})_{2}\right](9)$}

The steps of synthesis followed the procedure for compound 7. Reagents: $3-\left(\mathrm{PhCH}_{2} \mathrm{OCO}\right) \mathrm{C}_{9} \mathrm{H}_{7}(4 ; 0.50 \mathrm{~g}, 2 \mathrm{mmol}), 1.3 \mathrm{~mL}$ of ${ }^{n} \mathrm{BuLi}\left(1.6 \mathrm{~mol} \mathrm{~L}{ }^{-1}\right),\left[\left(\eta^{3}-\mathrm{C}_{3} \mathrm{H}_{5}\right) \mathrm{Mo}(\mathrm{CO})_{2}(\mathrm{NCMe})_{2} \mathrm{Cl}\right](6 ; 0.62 \mathrm{~g}$, $2 \mathrm{mmol})$. Yield: $0.60 \mathrm{~g}(1.36 \mathrm{mmol}, 68 \%)$. Yellow crystals. $\mathrm{Mp}=$ $101{ }^{\circ} \mathrm{C}$. Anal. Calc. for $\mathrm{C}_{22} \mathrm{H}_{18} \mathrm{MoO}_{4}$ : C: 59.74; $\mathrm{H}: 4.10$. Found: $\mathrm{C}$ : 59.79; H: 4.16. ${ }^{1} \mathrm{H}$ NMR $\left(\mathrm{CDCl}_{3}, 400 \mathrm{MHz}, \delta\right.$ ppm; $3: 1$ mixture of 9a $\left(\right.$ exo- $\left.\mathrm{C}_{3} \mathrm{H}_{5}\right)$ and $9 \mathbf{b}\left(\right.$ endo- $\left.\left.\mathrm{C}_{3} \mathrm{H}_{5}\right)\right)$ : 7.86-7.75 (m, $1 \mathrm{H}$ of $\mathbf{a}$ and $1 \mathrm{H}$ of $\mathbf{b}, \mathrm{H}^{4,7}$ of $\mathrm{C}_{9} \mathrm{H}_{6}$ ), 7.52-7.00 (m, $9 \mathrm{H}$ of $\mathbf{a}$ and $9 \mathrm{H}$ of $\mathbf{b}, \mathrm{H}^{4-7}$ of $\mathrm{C}_{9} H_{6}, \mathrm{C}_{6} H_{5}$ ), 6.25 (s-br, $1 \mathrm{H}$ of $\mathbf{a}, \mathrm{H}^{2,3}$ of $\mathrm{C}_{9} H_{6}$ ), 6.16 (s-br, $1 \mathrm{H}$ of $\mathbf{b}$, $\mathrm{H}^{2,3}$ of $\mathrm{C}_{9} H_{6}$ ), 6.03 (s-br, $1 \mathrm{H}$ of a, $\mathrm{H}^{2,3}$ of $\mathrm{C}_{9} H_{6}$ ), 5.99 (s-br, $1 \mathrm{H}$ of $\mathbf{b}, \mathrm{H}^{2,3}$ of $\left.\mathrm{C}_{9} H_{6}\right), 5.40\left(\mathrm{~m}, 2 \mathrm{H}\right.$ of $\mathbf{a}$ and $2 \mathrm{H}$ of $\left.\mathbf{b}, \mathrm{CH}_{2}\right), 3.44$ (s-br, $1 \mathrm{H}$ of $\mathbf{b}, \mathrm{C}_{3} H_{5}$ ), 3.29 (s-br, $2 \mathrm{H}$ of $\mathbf{b}, \mathrm{C}_{3} H_{5}$ ), 2.26 (s-br, $1 \mathrm{H}$ of a, syn of $\mathrm{C}_{3} \mathrm{H}_{5}$ ), 2.12 (s-br, $1 \mathrm{H}$ of a, syn of $\mathrm{C}_{3} \mathrm{H}_{5}$ ), 0.99 (s-br, $1 \mathrm{H}$ of a, $\left.\mathrm{C}_{3} H_{5}\right), 0.81\left(\mathrm{~s}-\mathrm{br}, 2 \mathrm{H}\right.$ of $\left.\mathbf{a}, \mathrm{C}_{3} H_{5}\right),-0.03(\mathrm{~s}-\mathrm{br}, 1 \mathrm{H}$ of $\mathbf{b}$, anti of $\mathrm{C}_{3} H_{5}$ ), -1.19 (s-br, $1 \mathrm{H}$ of $\mathbf{b}$, anti of $\mathrm{C}_{3} \mathrm{H}_{5}$ ). IR (ATR, $\mathrm{cm}^{-1}$ ): 1932 vs $\left[\nu_{\mathrm{a}}(\mathrm{CO})_{\mathrm{C} \equiv \mathrm{o}}\right], 1865 \mathrm{vs}\left[\nu_{\mathrm{s}}(\mathrm{CO})_{\mathrm{C} \equiv \mathrm{o}}\right], 1702 \mathrm{~s}\left[\nu(\mathrm{CO})_{\mathrm{C}=\mathrm{O}}\right]$. Raman (capillary, $\mathrm{cm}^{-1}$ ): 1942(6) $\left[\nu_{\mathrm{a}}(\mathrm{CO})_{\mathrm{C} \equiv \mathrm{O}}\right], 1856(10)$ $\left[\nu_{\mathrm{s}}(\mathrm{CO})_{\mathrm{C} \equiv \mathrm{o}}\right], 1705(5)\left[\nu(\mathrm{CO})_{\mathrm{C}=\mathrm{O}}\right]$.

\section{Synthesis of $\left[\left(\eta^{3}-\mathrm{C}_{3} \mathrm{H}_{5}\right)\left\{\eta^{5}-1-\left(4-\mathrm{MeOC}_{6} \mathrm{H}_{4} \mathrm{OCO}\right) \mathrm{C}_{9} \mathrm{H}_{6}\right\} \mathrm{Mo}(\mathrm{CO})_{2}\right](10)$}

The steps of synthesis followed the procedure for compound 7 . Reagents: 3-(4- $\left.\mathrm{MeOC}_{6} \mathrm{H}_{4} \mathrm{OCO}\right) \mathrm{C}_{9} \mathrm{H}_{7}(5 ; 0.53 \mathrm{~g}, 2 \mathrm{mmol}), 1.3 \mathrm{~mL}$ of ${ }^{n} \mathrm{BuLi}\left(1.6 \mathrm{~mol} \mathrm{~L}^{-1}\right),\left[\left(\eta^{3}-\mathrm{C}_{3} \mathrm{H}_{5}\right) \mathrm{Mo}(\mathrm{CO})_{2}(\mathrm{NCMe})_{2} \mathrm{Cl}\right](6 ; 0.62 \mathrm{~g}$, $2 \mathrm{mmol})$. Yield: $0.25 \mathrm{~g}(0.55 \mathrm{mmol}, 27 \%)$. Orange crystals. $\mathrm{Mp}=$ $124{ }^{\circ} \mathrm{C}$. Anal. Calc. for $\mathrm{C}_{22} \mathrm{H}_{18} \mathrm{MoO}_{5}$ : C: 57.65; H: 3.96. Found: C: 57.52; H: 3.80. ${ }^{1} \mathrm{H}$ NMR $\left(\mathrm{CDCl}_{3}, 400 \mathrm{MHz}, \delta \mathrm{ppm} ; 3: 1\right.$ mixture of 10a $\left(\right.$ exo- $\left.\mathrm{C}_{3} \mathrm{H}_{5}\right)$ and 10b $\left(\right.$ endo- $\left.\left.\mathrm{C}_{3} \mathrm{H}_{5}\right)\right): 7.90-7.80(\mathrm{~m}, 1 \mathrm{H}$ of a and $1 \mathrm{H}$ of $\mathbf{b}, \mathrm{H}^{4,7}$ of $\left.\mathrm{C}_{9} H_{6}\right), 7.30-6.90(\mathrm{~m}, 7 \mathrm{H}$ of $\mathbf{a}$ and $7 \mathrm{H}$ of $\mathbf{b}$, $\mathrm{H}^{4-7}$ of $\mathrm{C}_{9} \mathrm{H}_{6}$ and $\left.\mathrm{C}_{6} \mathrm{H}_{4}\right), 6.37$ (s-br, $1 \mathrm{H}$ of $\mathbf{a}, \mathrm{H}^{2,3}$ of $\mathrm{C}_{9} \mathrm{H}_{6}$ ), 6.29 (s-br, $1 \mathrm{H}$ of $\mathbf{b}, \mathrm{H}^{2,3}$ of $\mathrm{C}_{9} H_{6}$ ), 6.13 (s-br, $1 \mathrm{H}$ of $\mathbf{a}, \mathrm{H}^{2,3}$ of $\mathrm{C}_{9} H_{6}$ ), 6.06 (s-br, $1 \mathrm{H}$ of $\mathbf{b}, \mathrm{H}^{2,3}$ of $\left.\mathrm{C}_{9} H_{6}\right), 3.82(\mathrm{~s}, 3 \mathrm{H}$ of $\mathbf{a}$ and $3 \mathrm{H}$ of $\mathbf{b}$, $\mathrm{CH}_{3}$ ), 3.58 (s-br, $1 \mathrm{H}$ of $\mathbf{b}, \mathrm{C}_{3} H_{5}$ ), 3.35 (s-br, $2 \mathrm{H}$ of $\left.\mathbf{b}, \mathrm{C}_{3} H_{5}\right), 2.40$ $\left(\mathrm{d},{ }^{3} J\left({ }^{1} \mathrm{H},{ }^{1} \mathrm{H}\right)=6.0 \mathrm{~Hz}, 1 \mathrm{H}\right.$ of $\mathbf{a}$, syn of $\left.\mathrm{C}_{3} H_{5}\right), 2.24\left(\mathrm{~d},{ }^{3} J\left({ }^{1} \mathrm{H},{ }^{1} \mathrm{H}\right)=\right.$ $6.1 \mathrm{~Hz}, 1 \mathrm{H}$ of a, syn of $\left.\mathrm{C}_{3} H_{5}\right), 1.13\left(\mathrm{~d},{ }^{3} J\left({ }^{1} \mathrm{H},{ }^{1} \mathrm{H}\right)=11.0 \mathrm{~Hz}, 1 \mathrm{H}\right.$ of a, anti of $\left.\mathrm{C}_{3} \mathrm{H}_{5}\right), 0.89\left(\mathrm{~d},{ }^{3} J\left({ }^{1} \mathrm{H},{ }^{1} \mathrm{H}\right)=11.0 \mathrm{~Hz}, 1 \mathrm{H}\right.$ of a, anti of $\left.\mathrm{C}_{3} H_{5}\right), 0.63\left(\mathrm{~m}, 1 \mathrm{H}\right.$ of a, meso of $\left.\mathrm{C}_{3} H_{5}\right), 0.01\left(\mathrm{~d},{ }^{3} J\left({ }^{1} \mathrm{H},{ }^{1} \mathrm{H}\right)=\right.$ $9.5 \mathrm{~Hz}, 1 \mathrm{H}$ of $\mathbf{b}$, anti of $\left.\mathrm{C}_{3} H_{5}\right),-1.13\left(\mathrm{~d},{ }^{3} J\left({ }^{1} \mathrm{H},{ }^{1} \mathrm{H}\right)=9.6 \mathrm{~Hz}, 1 \mathrm{H}\right.$ of $\mathbf{b}$, anti of $\left.\mathrm{C}_{3} H_{5}\right)$. IR (ATR, $\left.\mathrm{cm}^{-1}\right)$ : 1944vs $\left[\nu_{\mathrm{a}}(\mathrm{CO})_{\mathrm{C} \equiv \mathrm{o}}\right], 1865 \mathrm{vs}$ $\left[\nu_{\mathrm{s}}(\mathrm{CO})_{\mathrm{C} \equiv \mathrm{O}}\right], 1722 \mathrm{~s} \quad\left[\nu(\mathrm{CO})_{\mathrm{C}=\mathrm{O}}\right]$. Raman (capillary, $\mathrm{cm}^{-1}$ ): $1946(3)\left[\nu_{\mathrm{a}}(\mathrm{CO})_{\mathrm{C} \equiv \mathrm{o}}\right], 1862(7)\left[\nu_{\mathrm{s}}(\mathrm{CO})_{\mathrm{C} \equiv \mathrm{O}}\right], 1714(9)\left[\nu(\mathrm{CO})_{\mathrm{C}=\mathrm{O}}\right]$.

\section{Synthesis of $\left[\left\{\eta^{3}-1-(M e O C O) C_{9} H_{6}\right\}\left(\eta^{5}-\right.\right.$ Ind $\left.) M o(C O)_{2}\right](12)$}

3-(MeOCO) $\mathrm{C}_{9} \mathrm{H}_{7}(2 ; 0.17 \mathrm{~g}, 1 \mathrm{mmol})$ was dissolved with $20 \mathrm{~mL}$ of THF, cooled at $0{ }^{\circ} \mathrm{C}$ and treated dropwise with $0.6 \mathrm{~mL}$ of ${ }^{n} \mathrm{BuLi}$ $\left(1.6 \mathrm{~mol} \mathrm{~L}^{-1}\right)$. The reaction mixture was stirred for $1 \mathrm{~h}$ and then added dropwise to the THF solution of $\left[\left\{\left(\eta^{5}\right.\right.\right.$-Ind $) \mathrm{Mo}(\mathrm{CO})_{2}$ $\left.(\mu-\mathrm{Cl})\}_{2}\right](11 ; 0.30 \mathrm{~g}, 0.5 \mathrm{mmol})$ precooled to $-80{ }^{\circ} \mathrm{C}$. The reaction mixture was stirred at room temperature overnight and then vacuum evaporated to dryness. The solid residue was washed with cold hexane $(10 \mathrm{~mL})$ and extracted several times with diethyl ether. The volatiles were vacuum evaporated and product was vacuum dried. Yield: $0.19 \mathrm{~g}$ (0.43 mmol, 43\%). Port red solid. $\mathrm{Mp}=120{ }^{\circ} \mathrm{C}$ (dec). Anal. Calc. for $\mathrm{C}_{22} \mathrm{H}_{18} \mathrm{MoO}_{4}$ : C: $59.74 ; \mathrm{H}: 4.10$. Found: C: 59.87; $\mathrm{H}: 4.03 .{ }^{1} \mathrm{H} \mathrm{NMR}\left(\mathrm{CDCl}_{3}\right.$; $400 \mathrm{MHz} ; \delta \mathrm{ppm}): 7.52-6.65\left(\mathrm{~m}, 9 \mathrm{H}, \mathrm{C}_{9} H_{6}\right.$ and $\left.\mathrm{C}_{9} H_{7}\right), 5.60$ $\left(\mathrm{m}, 3 \mathrm{H}, \mathrm{C}_{9} H_{6}\right.$ and $\left.\mathrm{C}_{9} H_{7}\right), 4.73\left(\mathrm{~s}-\mathrm{br}, 1 \mathrm{H}, \mathrm{C}_{9} H_{7}\right), 3.84(\mathrm{~s}, 3 \mathrm{H}$, $\left.\left(\mathrm{s}, 3 \mathrm{H}, \mathrm{COOCH} H_{3}\right)\right)$ IR $\left(\mathrm{ATR}, \mathrm{cm}^{-1}\right)$ : 1943vs $\left[\nu_{\mathrm{a}}(\mathrm{CO})_{\mathrm{C} \equiv \mathrm{o}}\right], 1876 \mathrm{vs}$ $\left[\nu_{\mathrm{s}}(\mathrm{CO})_{\mathrm{C} \equiv \mathrm{o}}\right], 1828 \mathrm{~s} \quad\left[\nu_{\mathrm{s}}(\mathrm{CO})_{\mathrm{C} \equiv \mathrm{o}}\right], 1694 \mathrm{~s} \quad\left[\nu(\mathrm{CO})_{\mathrm{C}=\mathrm{o}}\right]$. Single crystals suitable for X-ray diffraction analysis were obtained by recrystallization of the product from hot hexane.

\section{Synthesis of 3-(MeCO) $\mathrm{C}_{9} \mathrm{H}_{7}$ (13)}

The steps of synthesis followed the procedure for compound 2 . Reagents: indene $(\mathbf{1} ; 11.7 \mathrm{~mL}, 0.10 \mathrm{~mol})$, sodium hydride $(2.4 \mathrm{~g}$; $0.10 \mathrm{~mol})$, ethyl acetate $(9.8 \mathrm{~mL}, 0.10 \mathrm{~mol})$. The crude product was vacuum distilled $\left(90{ }^{\circ} \mathrm{C}, 660 \mathrm{~Pa}\right)$. Yield: $6.0 \mathrm{~g}(38 \mathrm{mmol}$, $38 \%$ ). Pale yellow solid. $\mathrm{Mp}=58{ }^{\circ} \mathrm{C}$. Anal. Calc. for $\mathrm{C}_{11} \mathrm{H}_{10} \mathrm{O}$ : C: 83.52; $\mathrm{H}: 6.37$. Found: C: 83.44; $\mathrm{H}: 6.31 .{ }^{1} \mathrm{H} \mathrm{NMR}\left(\mathrm{CDCl}_{3}\right.$; $400 \mathrm{MHz} ; \delta$ ppm): $8.19\left(\mathrm{~d},{ }^{3} J\left({ }^{1} \mathrm{H},{ }^{1} \mathrm{H}\right)=7.7 \mathrm{~Hz}, 1 \mathrm{H}, \mathrm{C}_{9} H_{7}\right), 7.41$ $\left(\mathrm{d},{ }^{3} J\left({ }^{1} \mathrm{H},{ }^{1} \mathrm{H}\right)=7.4 \mathrm{~Hz}, 1 \mathrm{H}, \mathrm{C}_{9} H_{7}\right), 7.30\left(\mathrm{t},{ }^{3} J\left({ }^{1} \mathrm{H},{ }^{1} \mathrm{H}\right)=7.4 \mathrm{~Hz}, 1 \mathrm{H}\right.$, $\left.\mathrm{C}_{9} H_{7}\right), 7.27\left(\mathrm{t},{ }^{3} J\left({ }^{1} \mathrm{H},{ }^{1} \mathrm{H}\right)=1.8 \mathrm{~Hz}, 1 \mathrm{H}, \mathrm{C}_{9} H_{7}\right), 7.22\left(\mathrm{t},{ }^{3} J\left({ }^{1} \mathrm{H},{ }^{1} \mathrm{H}\right)=\right.$ $\left.7.2 \mathrm{~Hz}, 1 \mathrm{H}, \mathrm{C}_{9} H_{7}\right), 3.47\left(\mathrm{~d},{ }^{3} J\left({ }^{1} \mathrm{H},{ }^{1} \mathrm{H}\right)=1.8 \mathrm{~Hz}, 2 \mathrm{H}, \mathrm{C}_{9} H_{7}\right), 2.46$ $\left(\mathrm{s}, 3 \mathrm{H}, \mathrm{COCH}_{3}\right) .{ }^{13} \mathrm{C}\left\{{ }^{1} \mathrm{H}\right\} \mathrm{NMR}\left(\mathrm{CDCl}_{3} ; 101 \mathrm{MHz} ; \delta \mathrm{ppm}\right): 196.2$ $\left(1 \mathrm{C}, \mathrm{C}_{\mathrm{q}}, C \mathrm{OMe}\right), 145.0\left(1 \mathrm{C}, \mathrm{CH}, C_{9} \mathrm{H}_{7}\right), 143.5,143.2,140.8$ 
$\left(3 \times 1 \mathrm{C}, \mathrm{C}_{\mathrm{q}}, C_{9} \mathrm{H}_{7}\right), 126.7,125.8,123.7,123.5\left(4 \times 1 \mathrm{C}, \mathrm{CH}, C_{9} \mathrm{H}_{7}\right)$, $38.6\left(1 \mathrm{C}, \mathrm{CH}_{2}, C_{9} \mathrm{H}_{7}\right), 27.7\left(1 \mathrm{C}, \mathrm{CH}_{3}, \mathrm{COCH}_{3}\right)$. IR (ATR, $\mathrm{cm}^{-1}$ ): 1669s $\left[\nu(\mathrm{CO})_{\mathrm{C}=\mathrm{O}}\right]$. Raman (capillary, $\left.\mathrm{cm}^{-1}\right)$ : 1666 $(8)\left[\nu(\mathrm{CO})_{\mathrm{C}=\mathrm{O}}\right]$. Single crystals suitable for X-ray diffraction analysis were obtained by recrystallization of the product from hexane.

\section{Synthesis of $\left[\left(\eta^{3}-\mathrm{C}_{3} \mathrm{H}_{5}\right)\left\{\eta^{5}-1-(\mathrm{MeCO}) \mathrm{C}_{9} \mathrm{H}_{6}\right\} \mathrm{Mo}(\mathrm{CO})_{2}\right](14)$}

The steps of synthesis followed the procedure for compound 7 . Reagents: 3-(MeCO) $\mathrm{C}_{9} \mathrm{H}_{7}$ (13; $\left.0.79 \mathrm{~g}, 5 \mathrm{mmol}\right), 3.1 \mathrm{~mL}$ of ${ }^{n} \mathrm{BuLi}$ $\left(1.6 \mathrm{~mol} \mathrm{~L}^{-1}\right),\left[\left(\eta^{3}-\mathrm{C}_{3} \mathrm{H}_{5}\right) \mathrm{Mo}(\mathrm{CO})_{2}(\mathrm{NCMe})_{2} \mathrm{Cl}\right](6 ; 1.55 \mathrm{~g}, 5 \mathrm{mmol})$. Yield: $1.1 \mathrm{~g}$ (3.1 mmol, 63\%). Yellow powder. $\mathrm{Mp}=124{ }^{\circ} \mathrm{C}$. Anal. Calc. for $\mathrm{C}_{16} \mathrm{H}_{14} \mathrm{MoO}_{3}$ : C: 54.78; H: 4.03. Found: C: $54.91 ; \mathrm{H}$ : 4.16. ${ }^{1} \mathrm{H}$ NMR $\left(\mathrm{CDCl}_{3}, 400 \mathrm{MHz}, \delta \mathrm{ppm} ; 3: 1\right.$ mixture of 14a $\left(\right.$ exo- $\left.\mathrm{C}_{3} \mathrm{H}_{5}\right)$ and $14 \mathbf{b}\left(\right.$ endo- $\left.\left.\mathrm{C}_{3} \mathrm{H}_{5}\right)\right): 8.05-7.98(\mathrm{~m}, 1 \mathrm{H}$ of $\mathbf{a}$ and $1 \mathrm{H}$ of $\mathbf{b}, \mathrm{H}^{4,7}$ of $\left.\mathrm{C}_{9} \mathrm{H}_{6}\right), 7.32-7.05\left(\mathrm{~m}, 3 \mathrm{H}\right.$ of $\mathbf{a}$ and $3 \mathrm{H}$ of $\mathbf{b}, \mathrm{H}^{4-7}$ of $\mathrm{C}_{9} H_{6}$ ), 6.05 (s-br, $2 \mathrm{H}$ of a and $2 \mathrm{H}$ of $\mathbf{b}, \mathrm{H}^{2,3}$ of $\mathrm{C}_{9} H_{6}$ ), 3.50 (s-br, $1 \mathrm{H}$ of $\left.\mathbf{b}, \mathrm{C}_{3} H_{5}\right), 3.29\left(\mathrm{~s}-\mathrm{br}, 2 \mathrm{H}\right.$ of $\left.\mathbf{b}, \mathrm{C}_{3} H_{5}\right), 2.54(\mathrm{~s}, 3 \mathrm{H}$ of $\mathbf{a}$ and $3 \mathrm{H}$ of $\mathbf{b}, \mathrm{CH}_{3}$ ), 2.25 (s-br, $1 \mathrm{H}$ of $\mathbf{a}$, syn of $\mathrm{C}_{3} \mathrm{H}_{5}$ ), 2.14 (s-br, $1 \mathrm{H}$ of $\mathbf{a}$, syn of $\left.\mathrm{C}_{3} \mathrm{H}_{5}\right), 1.09\left(\mathrm{~d},{ }^{3} \mathrm{~J}\left({ }^{1} \mathrm{H},{ }^{1} \mathrm{H}\right)=9.3 \mathrm{~Hz}, 1 \mathrm{H}\right.$ of a, anti of $\left.\mathrm{C}_{3} \mathrm{H}_{5}\right)$, $0.85\left(\mathrm{~d},{ }^{3} J\left({ }^{1} \mathrm{H},{ }^{1} \mathrm{H}\right)=9.5 \mathrm{~Hz}, 1 \mathrm{H}\right.$ of a, anti of $\left.\mathrm{C}_{3} H_{5}\right), 0.64(\mathrm{~s}-\mathrm{br}, 1 \mathrm{H}$ of a, meso of $\mathrm{C}_{3} \mathrm{H}_{5}$ ), 0.06 (s-br, $1 \mathrm{H}$ of $\mathbf{b}$, anti of $\mathrm{C}_{3} \mathrm{H}_{5}$ ), -1.33 (s-br, $1 \mathrm{H}$ of $\mathbf{b}$, anti of $\left.\mathrm{C}_{3} H_{5}\right)$. IR (ATR, $\left.\mathrm{cm}^{-1}\right): 1936 \mathrm{vs}\left[\nu_{\mathrm{a}}(\mathrm{CO})_{\mathrm{C} \equiv \mathrm{o}}\right.$, 1859vs $\left[\nu_{\mathrm{S}}(\mathrm{CO})_{\mathrm{C} \equiv \mathrm{o}}\right], 1660 \mathrm{~s} \quad\left[\nu(\mathrm{CO})_{\mathrm{C}=\mathrm{O}}\right]$. Raman (capillary, $\left.\mathrm{cm}^{-1}\right): 1932(5)\left[\nu_{\mathrm{a}}(\mathrm{CO})_{\mathrm{C} \equiv \mathrm{o}}\right], 1836(10)\left[\nu_{\mathrm{s}}(\mathrm{CO})_{\mathrm{C} \equiv \mathrm{o}}\right], 1666(7)$ $\left[\nu(\mathrm{CO})_{\mathrm{C}=\mathrm{O}}\right]$. Single crystals suitable for X-ray diffraction analysis were obtained by recrystallization of the product from hexane.

\section{Synthesis of 3-( ${ }^{t} \mathrm{BuNHCO} \mathrm{C}_{9} \mathrm{H}_{7}$ (15)}

Lithium indenide (1-Li), freshly prepared from indene $(\mathbf{1} ; \mathbf{1 1 . 7} \mathrm{mL}$, $0.10 \mathrm{~mol})$ and $62.5 \mathrm{~mL}$ of ${ }^{n} \mathrm{BuLi}\left(1.6 \mathrm{~mol} \mathrm{~L}^{-1}\right)$ in THF $(150 \mathrm{~mL})$, was cooled at $-80{ }^{\circ} \mathrm{C}$, treated with ${ }^{t}$ BuNCO (11.4 mL, $0.10 \mathrm{~mol}$ ), slowly warmed at room temperature and stirred overnight. The reaction mixture was poured on an ice/water mixture. The pale yellow precipitate was filtered on a glass frit and washed with hexane. The crude product was dissolved in $\mathrm{CH}_{2} \mathrm{Cl}_{2}$ and water. The organic phase was separated, dried with magnesium sulfate and volatiles were evaporated on a rotavapor. The final product was washed with hexane on a glass frit and vacuum dried. Yield: $14.0 \mathrm{~g}$ (65 mmol, 65\%). Colorless crystals. $\mathrm{Mp}=122{ }^{\circ} \mathrm{C}$. Anal. Calc. for $\mathrm{C}_{14} \mathrm{H}_{17} \mathrm{NO}$ : C: 78.10; $\mathrm{H}: 7.96$; N: 6.51. Found: $\mathrm{C}$ : 78.22; $\mathrm{H}: 8.04$; $\mathrm{N}: 6.67 .{ }^{1} \mathrm{H}$ NMR $\left(\mathrm{CDCl}_{3} ; 400 \mathrm{MHz} ; \delta \mathrm{ppm}\right): 7.89\left(\mathrm{~d},{ }^{3} J\left({ }^{1} \mathrm{H},{ }^{1} \mathrm{H}\right)=\right.$ $\left.7.7 \mathrm{~Hz}, 1 \mathrm{H}, \mathrm{C}_{9} H_{7}\right), 7.49\left(\mathrm{~d},{ }^{3} J\left({ }^{1} \mathrm{H},{ }^{1} \mathrm{H}\right)=7.4 \mathrm{~Hz}, 1 \mathrm{H}, \mathrm{C}_{9} H_{7}\right), 7.36$ $\left(\mathrm{t},{ }^{3} J\left({ }^{1} \mathrm{H},{ }^{1} \mathrm{H}\right)=7.5 \mathrm{~Hz}, 1 \mathrm{H}, \mathrm{C}_{9} H_{7}\right), 7.28\left(\mathrm{t},{ }^{3} J\left({ }^{1} \mathrm{H},{ }^{1} \mathrm{H}\right)=7.4 \mathrm{~Hz}, 1 \mathrm{H}\right.$, $\left.\mathrm{C}_{9} H_{7}\right), 6.90\left(\mathrm{~s}, 1 \mathrm{H}, \mathrm{C}_{9} H_{7}\right), 5.89\left(\mathrm{~s}, 1 \mathrm{H}, \mathrm{N} H^{t} \mathrm{Bu}\right), 3.48\left(\mathrm{~s}, 2 \mathrm{H}, \mathrm{C}_{9} H_{7}\right)$, 1.51 (s, 9H, C(CH3) $\left.)_{3}\right) .{ }^{13} \mathrm{C}\left\{{ }^{1} \mathrm{H}\right\}$ NMR $\left(\mathrm{CDCl}_{3} ; 101 \mathrm{MHz} ; \delta \mathrm{ppm}\right)$ : $164.7\left(1 \mathrm{C}, \mathrm{C}_{\mathrm{q}}, C \mathrm{ONH}\right), 143.9,141.6\left(2 \times 1 \mathrm{C}, \mathrm{C}_{\mathrm{q}}, C_{9} \mathrm{H}_{7}\right), 136.7$, 126.7, 125.7, 124.1, $121.9\left(5 \times 1 \mathrm{C}, \mathrm{CH}, C_{9} \mathrm{H}_{7}\right), 51.7\left(1 \mathrm{C}, \mathrm{C}_{\mathrm{q}}\right.$, $\left.C\left(\mathrm{CH}_{3}\right)_{3}\right), 38.2\left(1 \mathrm{C}, \mathrm{CH}_{2}, C_{9} \mathrm{H}_{7}\right), 29.1\left(3 \mathrm{C}, \mathrm{CH}_{3}, \mathrm{C}_{\mathrm{q}}, \mathrm{C}\left(\mathrm{CH}_{3}\right)_{3}\right)$. IR (ATR, $\left.\mathrm{cm}^{-1}\right): 3286 \mathrm{~m}[\nu(\mathrm{NH})], 1637 \mathrm{~s}\left[\nu(\mathrm{CO})_{\mathrm{C}=\mathrm{O}}\right]$. Raman (capillary, $\left.\mathrm{cm}^{-1}\right): 1638(8)\left[\nu(\mathrm{CO})_{\mathrm{C}=\mathrm{O}}\right]$.

\section{Synthesis of 3-( $\mathrm{PhNHCO} \mathrm{C}_{9} \mathrm{H}_{7}$ (16)}

3-(HOCO) $\mathrm{C}_{9} \mathrm{H}_{7}$ (0.80 g; $5.0 \mathrm{mmol}$ ) was dissolved in the excess of thionyl chloride $(5 \mathrm{~mL})$ and stirred at room temperature for $1 \mathrm{~h}$ and then heated at $60{ }^{\circ} \mathrm{C}$ for $10 \mathrm{~min}$. The volatiles were vacuum evaporated. The sticky solid was dissolved in $\mathrm{CH}_{2} \mathrm{Cl}_{2}$, cooled at
$-80{ }^{\circ} \mathrm{C}$, treated with the excess of $\mathrm{PhNH}_{2}(2.7 \mathrm{~mL}, 30 \mathrm{mmol})$ and stirred at room temperature for $2 \mathrm{~h}$. The volatiles were vacuum evaporated and the solid product was washed with diethyl ether. The crude product was purified by column chromatography on silica (hexane/ethyl acetate $=7: 3$ ). Yield: $0.62 \mathrm{~g}$ (2.6 mmol, 53\%). Colorless crystals. $\mathrm{Mp}=155^{\circ} \mathrm{C}$. $\mathrm{R}_{\mathrm{f}}(\mathrm{TLC}$; hexane/ethyl acetate $=7: 3)=0.44$. Anal. Calc. for $\mathrm{C}_{16} \mathrm{H}_{13} \mathrm{NO}$ : C: 81.68; H: 5.57; N: 5.95. Found: 81.52; H: 5.55; N: 5.84. ${ }^{1} \mathrm{H}$ NMR $\left(\mathrm{CDCl}_{3} ; 400 \mathrm{MHz} ; \delta \mathrm{ppm}\right): 7.94\left(\mathrm{~d},{ }^{3} J\left({ }^{1} \mathrm{H},{ }^{1} \mathrm{H}\right)=7.7 \mathrm{~Hz}, 1 \mathrm{H}, \mathrm{C}_{9} H_{7}\right)$, $7.81(\mathrm{~s}, 1 \mathrm{H}, \mathrm{N} H \mathrm{Ph}), 7.63\left(\mathrm{~d},{ }^{3} J\left({ }^{1} \mathrm{H},{ }^{1} \mathrm{H}\right)=8.6 \mathrm{~Hz}, 2 \mathrm{H}, \mathrm{C}_{6} H_{5}\right), 7.48$ $\left(\mathrm{d},{ }^{3} J\left({ }^{1} \mathrm{H},{ }^{1} \mathrm{H}\right)=7.4 \mathrm{~Hz}, 1 \mathrm{H}, \mathrm{C}_{9} H_{7}\right), 7.37-7.32\left(\mathrm{~m}, 1 \mathrm{H}\right.$ of $\mathrm{C}_{9} H_{7}$ and $2 \mathrm{H}$ of $\left.\mathrm{C}_{6} H_{5}\right), 7.27\left(\mathrm{td},{ }^{3} J\left({ }^{1} \mathrm{H},{ }^{1} \mathrm{H}\right)=7.4 \mathrm{~Hz},{ }^{4} J\left({ }^{1} \mathrm{H},{ }^{1} \mathrm{H}\right)=1.2 \mathrm{~Hz}, 1 \mathrm{H}\right.$, $\left.\mathrm{C}_{9} H_{7}\right), 7.13\left(\mathrm{tt},{ }^{3} J\left({ }^{1} \mathrm{H},{ }^{1} \mathrm{H}\right)=7.5 \mathrm{~Hz},{ }^{4} J\left({ }^{1} \mathrm{H},{ }^{1} \mathrm{H}\right)=1.0 \mathrm{~Hz}, 1 \mathrm{H}, \mathrm{C}_{6} H_{5}\right)$, $7.06\left(\mathrm{t},{ }^{3} J\left({ }^{1} \mathrm{H},{ }^{1} \mathrm{H}\right)=2.0 \mathrm{~Hz}, 1 \mathrm{H}, \mathrm{C}_{9} H_{7}\right), 3.50\left(\mathrm{~d},{ }^{3} J\left({ }^{1} \mathrm{H},{ }^{1} \mathrm{H}\right)=2.0 \mathrm{~Hz}\right.$, $\left.2 \mathrm{H}, \mathrm{C}_{9} H_{7}\right) .{ }^{13} \mathrm{C}\left\{{ }^{1} \mathrm{H}\right\} \operatorname{NMR}\left(\mathrm{CDCl}_{3} ; 101 \mathrm{MHz} ; \delta \mathrm{ppm}\right): 163.3$ (1C, $\left.\mathrm{C}_{\mathrm{q}}, C \mathrm{ONH}\right), 143.7,141.2,140.9,137.9\left(4 \times 1 \mathrm{C}, \mathrm{C}_{\mathrm{q}}, C_{9} \mathrm{H}_{7}\right.$ and $\left.C_{6} \mathrm{H}_{5}\right), 137.0\left(1 \mathrm{C}, \mathrm{CH}, C_{9} \mathrm{H}_{7}\right), 129.3\left(2 \mathrm{C}, \mathrm{CH}, C_{6} \mathrm{H}_{5}\right), 127.0$, $126.0\left(2 \times 1 \mathrm{C}, \mathrm{CH}, C_{9} \mathrm{H}_{7}\right), 124.7\left(2 \mathrm{C}, \mathrm{CH}, C_{6} \mathrm{H}_{5}\right), 124.1,122.2$ $\left(2 \times 1 \mathrm{C}, \mathrm{CH}, C_{9} \mathrm{H}_{7}\right), 120.3\left(2 \mathrm{C}, \mathrm{CH}, C_{6} \mathrm{H}_{5}\right), 38.6\left(1 \mathrm{C}, \mathrm{CH}_{2}, C_{9} \mathrm{H}_{7}\right)$. IR $\left(\right.$ ATR, $\left.\mathrm{cm}^{-1}\right)$ : 3284s $[\nu(\mathrm{NH})], 1648 \mathrm{~s}\left[\nu(\mathrm{CO})_{\mathrm{C}=\mathrm{O}}\right]$. Raman (capillary, $\left.\mathrm{cm}^{-1}\right)$ : $1650(9)\left[\nu(\mathrm{CO})_{\mathrm{C}=\mathrm{O}}\right]$.

\section{Reaction of 1-Na with PhNCO}

Lithium indenide (1-Li), freshly prepared from indene $(\mathbf{1} ; 11.7 \mathrm{~mL}$, $0.10 \mathrm{~mol})$ and $62.5 \mathrm{~mL}$ of ${ }^{n} \mathrm{BuLi}\left(1.6 \mathrm{~mol} \mathrm{~L}^{-1}\right)$ in THF $(150 \mathrm{~mL})$, was cooled at $-80{ }^{\circ} \mathrm{C}$, treated with PhNCO (10.9 mL, $0.10 \mathrm{~mol}$ ), slowly warmed at room temperature and stirred overnight. The reaction mixture was poured on an ice/water mixture, treated with $\mathrm{CH}_{2} \mathrm{Cl}_{2}$ and neutralized with hydrochloric acid. The aqueous phase was separated and disposed. The organic phase was filtered on a glass frit. The collected solid was recrystallized from acetone to give colorless crystals of $\mathbf{1 8}$ (yield: $2.9 \mathrm{~g}, 8.2 \mathrm{mmol}, 8 \%$ ). The volatiles from the filtrate were vacuum evaporated and the product was washed with diethyl ether to give $9 \mathrm{~g}$ of a mixture of 16 and 17 in a molar ratio of 1:5 (according to ${ }^{1} \mathrm{H} \mathrm{NMR}$ ). Analytically pure samples of $\mathbf{1 6}$ and 17 were obtained by column chromatography on silica (hexane/ethyl acetate = $7: 3)$. 1,3-(PhNHCO) ${ }_{2} \mathrm{C}_{9} \mathrm{H}_{6}$ (18): white solid. $\mathrm{Mp}=240{ }^{\circ} \mathrm{C}$ (dec.). Anal. Calc. for $\mathrm{C}_{23} \mathrm{H}_{18} \mathrm{~N}_{2} \mathrm{O}_{2}$ : C: 77.95; $\mathrm{H}: 5.12 ; \mathrm{N}: 7.90$. Found: C: 77.90; H: 5.04; N: 7.96. ${ }^{1} \mathrm{H}$ NMR (acetone- $d^{6}$; $400 \mathrm{MHz} \delta \mathrm{ppm}): 9.66$ (s, 1H, NHPh), 9.14 (s, 1H, NHPh), $8.03\left(\mathrm{~d},{ }^{3} J\left({ }^{1} \mathrm{H},{ }^{1} \mathrm{H}\right)=7.6 \mathrm{~Hz}, 1 \mathrm{H}, \mathrm{C}_{9} H_{6}\right), 7.85\left(\mathrm{~d},{ }^{3} J\left({ }^{1} \mathrm{H},{ }^{1} \mathrm{H}\right)=8.2 \mathrm{~Hz}\right.$, $\left.2 \mathrm{H}, \mathrm{C}_{6} H_{5}\right), 7.68-7.63\left(\mathrm{~m}, 1 \mathrm{H}\right.$ of $\mathrm{C}_{9} H_{6}$ and $2 \mathrm{H}$ of $\left.\mathrm{C}_{6} H_{5}\right), 7.42-7.27$ $\left(\mathrm{m}, 3 \mathrm{H}\right.$ of $\mathrm{C}_{9} H_{6}$ and $4 \mathrm{H}$ of $\left.\mathrm{C}_{6} H_{5}\right), 7.12\left(\mathrm{tt},{ }^{3} \mathrm{~J}\left({ }^{1} \mathrm{H},{ }^{1} \mathrm{H}\right)=7.3 \mathrm{~Hz}\right.$, $\left.{ }^{4} J\left({ }^{1} \mathrm{H},{ }^{1} \mathrm{H}\right)=1.1 \mathrm{~Hz}, 1 \mathrm{H}, \mathrm{C}_{6} H_{5}\right), 7.08\left(\mathrm{tt},{ }^{3} J\left({ }^{1} \mathrm{H},{ }^{1} \mathrm{H}\right)=7.3 \mathrm{~Hz}\right.$, $\left.{ }^{4} J\left({ }^{1} \mathrm{H},{ }^{1} \mathrm{H}\right)=1.1 \mathrm{~Hz}, 1 \mathrm{H}, \mathrm{C}_{6} H_{5}\right), 4.71\left(\mathrm{~d},{ }^{3} J\left({ }^{1} \mathrm{H},{ }^{1} \mathrm{H}\right)=2.1 \mathrm{~Hz}, \mathrm{H}\right.$, $\left.\mathrm{C}_{9} H_{6}\right)$. IR (ATR, $\mathrm{cm}^{-1}$ ): 3236m [ $\left.\nu(\mathrm{NH})\right], 1662 \mathrm{~s}\left[\nu(\mathrm{CO})_{\mathrm{C}=\mathrm{O}}\right], 1646 \mathrm{~s}$ $\left[\nu(\mathrm{CO})_{\mathrm{C}=\mathrm{O}}\right]$. Raman (capillary, $\left.\mathrm{cm}^{-1}\right):$ 1647(9) $\left[\nu(\mathrm{CO})_{\mathrm{C}=\mathrm{O}}\right]$. 3-(PhNHCONPhCO) $\mathrm{C}_{9} \mathrm{H}_{7}$ (17): colorless crystals. $\mathrm{Mp}=116{ }^{\circ} \mathrm{C}$. $\mathrm{R}_{\mathrm{f}}$ (TLC; hexane/ethyl acetate $\left.=7: 3\right)=0.53$. Anal. Calc. for $\mathrm{C}_{23} \mathrm{H}_{18} \mathrm{~N}_{2} \mathrm{O}_{2}$ : C: 77.95; H: 5.12; N: 7.90. Found: C: 77.84; H: 5.18; N: 7.79. ${ }^{1} \mathrm{H}$ NMR $\left(\mathrm{CDCl}_{3} ; 400 \mathrm{MHz} ; \delta \mathrm{ppm}\right): 11.60(\mathrm{~s}, 1 \mathrm{H}$, $\mathrm{N} H \mathrm{Ph}), 7.75\left(\mathrm{~d},{ }^{3} J\left({ }^{1} \mathrm{H},{ }^{1} \mathrm{H}\right)=7.7 \mathrm{~Hz}, 1 \mathrm{H}, \mathrm{C}_{9} H_{7}\right), 7.66\left(\mathrm{~d},{ }^{3} J\left({ }^{1} \mathrm{H},{ }^{1} \mathrm{H}\right)\right.$ $\left.=8.2 \mathrm{~Hz}, 2 \mathrm{H}, \mathrm{C}_{6} H_{5}\right), 7.43-7.25\left(\mathrm{~m}, 3 \mathrm{H}\right.$ of $\mathrm{C}_{9} H_{7}$ and $7 \mathrm{H}$ of $\left.\mathrm{C}_{6} H_{5}\right)$, $7.16\left(\mathrm{tt},{ }^{3} J\left({ }^{1} \mathrm{H},{ }^{1} \mathrm{H}\right)=7.4 \mathrm{~Hz},{ }^{4} J\left({ }^{1} \mathrm{H},{ }^{1} \mathrm{H}\right)=1.1 \mathrm{~Hz}, 1 \mathrm{H}, \mathrm{C}_{6} H_{5}\right), 6.07$ $\left(\mathrm{t},{ }^{3} J\left({ }^{1} \mathrm{H},{ }^{1} \mathrm{H}\right)=2.1 \mathrm{~Hz}, 1 \mathrm{H}, \mathrm{C}_{9} H_{7}\right), 3.25\left(\mathrm{~d},{ }^{3} J\left({ }^{1} \mathrm{H},{ }^{1} \mathrm{H}\right)=2.0 \mathrm{~Hz}, 2 \mathrm{H}\right.$, 
$\left.\mathrm{C}_{9} \mathrm{H}_{7}\right) .{ }^{13} \mathrm{C}\left\{{ }^{1} \mathrm{H}\right\}$ NMR $\left(\mathrm{CDCl}_{3} ; 101 \mathrm{MHz} ; \delta \mathrm{ppm}\right): 170.0,152.2$ $\left(2 \times 1 \mathrm{C}, \mathrm{C}_{\mathrm{q}}, C \mathrm{O}\right), 142.5,141.9,138.9,138.7,137.9\left(5 \times 1 \mathrm{C}, \mathrm{C}_{\mathrm{q}}\right.$, $C_{9} \mathrm{H}_{7}$ and $C_{6} \mathrm{H}_{5}$ ), 139.0 (1C, $\mathrm{CH}, C_{9} \mathrm{H}_{7}$ ), 129.8, 129.2, 129.1 $\left(3 \times 2 \mathrm{C}, \mathrm{CH}, C_{6} \mathrm{H}_{5}\right), 128.9\left(1 \mathrm{C}, \mathrm{CH}, C_{6} \mathrm{H}_{5}\right), 126.9,126.0(2 \times$ $\left.1 \mathrm{C}, \mathrm{CH}, C_{9} \mathrm{H}_{7}\right), 124.5,124.0\left(2 \times 1 \mathrm{C}, \mathrm{CH}, C_{9} \mathrm{H}_{7}\right.$ and $\left.C_{6} \mathrm{H}_{5}\right), 121.5$ $\left(1 \mathrm{C}, \mathrm{CH}, C_{9} \mathrm{H}_{7}\right), 120.6\left(2 \mathrm{C}, \mathrm{CH}, C_{6} \mathrm{H}_{5}\right), 39.2\left(1 \mathrm{C}, \mathrm{CH}_{2}, C_{9} \mathrm{H}_{7}\right)$. IR $\left(\right.$ ATR, $\left.\mathrm{cm}^{-1}\right): 3214 \mathrm{~m}[\nu(\mathrm{NH})], 1646 \mathrm{~s}\left[\nu(\mathrm{CO})_{\mathrm{C}=\mathrm{O}}\right]$. Raman (capillary, $\left.\mathrm{cm}^{-1}\right)$ : 1723(1) $\left[\nu(\mathrm{CO})_{\mathrm{C}=\mathrm{O}}\right], 1648(9)\left[\nu(\mathrm{CO})_{\mathrm{C}=\mathrm{O}}\right]$.

\section{Synthesis of 3-(4- $\left.\mathrm{ClC}_{6} \mathrm{H}_{4} \mathrm{NHCO}\right) \mathrm{C}_{9} \mathrm{H}_{7}(19)$}

The steps of synthesis followed the procedure for compound 16. Reagents: $3-(\mathrm{HOCO}) \mathrm{C}_{9} \mathrm{H}_{7}(0.80 \mathrm{~g}, 5.0 \mathrm{mmol}), 4-\mathrm{ClC}_{6} \mathrm{H}_{4} \mathrm{NH}_{2}$ $(2.6 \mathrm{~g}, 20 \mathrm{mmol})$. The crude product was washed with ether and purified by column chromatography on silica (hexane/ethyl acetate $=7: 3)$. Yield: $0.60 \mathrm{~g}(2.2 \mathrm{mmol}, 44 \%)$. Colorless crystals. $\mathrm{Mp}=172{ }^{\circ} \mathrm{C} . \mathrm{R}_{\mathrm{f}}(\mathrm{TLC}$; hexane/ethyl acetate $=7: 3)=0.42$. Anal. Calc. for $\mathrm{C}_{16} \mathrm{H}_{12} \mathrm{ClNO}$ : $\mathrm{C}$ 71.25; $\mathrm{H}: 4.48 ; \mathrm{N}: 5.19$. Found: $\mathrm{C}$ : 71.35; H: 4.46; N: 5.12. ${ }^{1} \mathrm{H}$ NMR $\left(\mathrm{CDCl}_{3} ; 400 \mathrm{MHz} ; \delta \mathrm{ppm}\right): 7.91$ $\left(\mathrm{d},{ }^{3} J\left({ }^{1} \mathrm{H},{ }^{1} \mathrm{H}\right)=7.5 \mathrm{~Hz}, 1 \mathrm{H}, \mathrm{C}_{9} H_{7}\right), 7.80(\mathrm{~s}, 1 \mathrm{H}, \mathrm{NHPh}), 7.57$ $\left(\mathrm{d},{ }^{3} J\left({ }^{1} \mathrm{H},{ }^{1} \mathrm{H}\right)=8.8 \mathrm{~Hz}, 2 \mathrm{H}, \mathrm{C}_{6} H_{4}\right), 7.48\left(\mathrm{~d},{ }^{3} J\left({ }^{1} \mathrm{H},{ }^{1} \mathrm{H}\right)=7.4 \mathrm{~Hz}, 1 \mathrm{H}\right.$, $\left.\mathrm{C}_{9} H_{7}\right), 7.34\left(\mathrm{t},{ }^{3} J\left({ }^{1} \mathrm{H},{ }^{1} \mathrm{H}\right)=7.5 \mathrm{~Hz}, 1 \mathrm{H}, \mathrm{C}_{9} H_{7}\right), 7.29\left(\mathrm{~d},{ }^{3} J\left({ }^{1} \mathrm{H},{ }^{1} \mathrm{H}\right)=\right.$ $\left.8.8 \mathrm{~Hz}, 2 \mathrm{H}, \mathrm{C}_{6} H_{4}\right), 7.27\left(\mathrm{td},{ }^{3} J\left({ }^{1} \mathrm{H},{ }^{1} \mathrm{H}\right)=7.4 \mathrm{~Hz},{ }^{4} J\left({ }^{1} \mathrm{H},{ }^{1} \mathrm{H}\right)=\right.$ $\left.1.1 \mathrm{~Hz}, 1 \mathrm{H}, \mathrm{C}_{9} H_{7}\right), 7.05\left(\mathrm{t},{ }^{3} J\left({ }^{1} \mathrm{H},{ }^{1} \mathrm{H}\right)=2.0 \mathrm{~Hz}, 1 \mathrm{H}, \mathrm{C}_{9} H_{7}\right), 3.50$ $\left(\mathrm{d},{ }^{3} J\left({ }^{1} \mathrm{H},{ }^{1} \mathrm{H}\right)=2.0 \mathrm{~Hz}, 2 \mathrm{H}, \mathrm{C}_{9} H_{7}\right) .{ }^{13} \mathrm{C}\left\{{ }^{1} \mathrm{H}\right\} \mathrm{NMR}\left(\mathrm{CDCl}_{3} ; 101\right.$ $\mathrm{MHz} \delta$ ppm): 163.2 (1C, $\left.\mathrm{C}_{\mathrm{q}}, C \mathrm{ONH}\right), 143.7,141.1,140.7,136.5$, $127.9\left(5 \times 1 \mathrm{C}, \mathrm{C}_{\mathrm{q}}, C_{9} \mathrm{H}_{7}\right.$ and $\left.C_{6} \mathrm{H}_{4}\right), 137.3\left(1 \mathrm{C}, \mathrm{CH}, C_{9} \mathrm{H}_{7}\right), 129.3$ $\left(2 \mathrm{C}, \mathrm{CH}, C_{6} \mathrm{H}_{5}\right), 127.0,126.2,124.2,122.1,\left(4 \times 1 \mathrm{C}, \mathrm{CH}, C_{9} \mathrm{H}_{7}\right)$, $121.6\left(2 \mathrm{C}, \mathrm{CH}, C_{6} \mathrm{H}_{5}\right), 38.6\left(1 \mathrm{C}, \mathrm{CH}_{2}, C_{9} \mathrm{H}_{7}\right)$. IR (ATR, $\left.\mathrm{cm}^{-1}\right)$ : $3284 \mathrm{~m}[\nu(\mathrm{NH})], 1655 \mathrm{~s}\left[\nu(\mathrm{CO})_{\mathrm{C}=\mathrm{O}}\right]$. Raman (capillary, $\left.\mathrm{cm}^{-1}\right)$ : $1657(10)\left[\nu(\mathrm{CO})_{\mathrm{C}=\mathrm{O}}\right]$.

\section{Synthesis of $\left[\left(\eta^{3}-\mathrm{C}_{3} \mathrm{H}_{5}\right)\left\{\eta^{5}-1-\left({ }^{t} \mathrm{BuNHCO}\right) \mathrm{C}_{9} \mathrm{H}_{6}\right\} \mathrm{Mo}(\mathrm{CO})_{2}\right](20)$}

The steps of synthesis followed the procedure for compound 7. Reagents: $3-\left({ }^{t} \mathrm{BuNHCO}\right) \mathrm{C}_{9} \mathrm{H}_{7}(\mathbf{1 5} ; 0.43 \mathrm{~g}, 2 \mathrm{mmol}), 1.3 \mathrm{~mL}$ of ${ }^{n} \mathrm{BuLi}\left(1.6 \mathrm{~mol} \mathrm{~L}^{-1}\right),\left[\left(\eta^{3}-\mathrm{C}_{3} \mathrm{H}_{5}\right) \mathrm{Mo}(\mathrm{CO})_{2}(\mathrm{NCMe})_{2} \mathrm{Cl}\right](6 ; 0.62 \mathrm{~g}$, $2 \mathrm{mmol})$. Yield: $0.50 \mathrm{~g}(1.23 \mathrm{mmol}, 61 \%)$. Yellow powder. $\mathrm{Mp}=$ $190{ }^{\circ} \mathrm{C}$ (dec.). Anal. Calc. for $\mathrm{C}_{19} \mathrm{H}_{21} \mathrm{MoNO}_{3}$ : C: 56.03; H: 5.20; $\mathrm{N}: 3.44$. Found: C: $56.18 ; \mathrm{H}: 5.33 ; \mathrm{N}: 3.51 .{ }^{1} \mathrm{H}$ NMR $\left(\mathrm{CDCl}_{3}\right.$, $400 \mathrm{MHz}, \delta \mathrm{ppm} ; 3: 1$ mixture of $20 \mathrm{a}\left(\right.$ exo- $\left.\mathrm{C}_{3} \mathrm{H}_{5}\right)$ and $20 \mathrm{~b}$ (endo$\left.\mathrm{C}_{3} \mathrm{H}_{5}\right)$ ): $8.00-7.91\left(\mathrm{~m}, 1 \mathrm{H}\right.$ of $\mathbf{a}$ and $1 \mathrm{H}$ of $\mathbf{b}, \mathrm{H}^{4,7}$ of $\left.\mathrm{C}_{9} H_{6}\right), 7.21-$ $6.98\left(\mathrm{~m}, 3 \mathrm{H}\right.$ of $\mathbf{a}$ and $3 \mathrm{H}$ of $\mathbf{b}, \mathrm{H}^{4-7}$ of $\left.\mathrm{C}_{9} H_{6}\right), 5.98\left(\mathrm{~d},{ }^{3} J\left({ }^{1} \mathrm{H},{ }^{1} \mathrm{H}\right)=\right.$ $3.0 \mathrm{~Hz}, 1 \mathrm{H}$ of $\mathbf{a}, \mathrm{H}^{2,3}$ of $\left.\mathrm{C}_{9} H_{6}\right), 5.91\left(\mathrm{~d},{ }^{3} J\left({ }^{1} \mathrm{H},{ }^{1} \mathrm{H}\right)=2.5 \mathrm{~Hz}, 1 \mathrm{H}\right.$ of b, $\mathrm{H}^{2,3}$ of $\left.\mathrm{C}_{9} H_{6}\right), 5.86\left(\mathrm{~d},{ }^{3} J\left({ }^{1} \mathrm{H},{ }^{1} \mathrm{H}\right)=3.0 \mathrm{~Hz}, 1 \mathrm{H}\right.$ of $\mathbf{a}, \mathrm{H}^{2,3}$ of $\left.\mathrm{C}_{9} H_{6}\right), 5.79\left(\mathrm{~d},{ }^{3} J\left({ }^{1} \mathrm{H},{ }^{1} \mathrm{H}\right)=2.5 \mathrm{~Hz}, 1 \mathrm{H}\right.$ of $\mathbf{b}, \mathrm{H}^{2,3}$ of $\left.\mathrm{C}_{9} H_{6}\right), 5.68$ (s, $1 \mathrm{H}$ of $\mathbf{a}, \mathrm{NH}), 5.62(\mathrm{~s}, 1 \mathrm{H}$ of $\mathbf{b}, \mathrm{NH}), 3.54$ (s-br, $1 \mathrm{H}$ of $\mathbf{b}, \mathrm{C}_{3} H_{5}$ ), 3.33 (s-br, $2 \mathrm{H}$ of b, $\left.\mathrm{C}_{3} H_{5}\right), 2.44\left(\mathrm{~d},{ }^{3} J\left({ }^{1} \mathrm{H},{ }^{1} \mathrm{H}\right)=6.7 \mathrm{~Hz}, 1 \mathrm{H}\right.$ of $\mathbf{a}$, syn of $\left.\mathrm{C}_{3} H_{5}\right), 2.11\left(\mathrm{~d},{ }^{3} J\left({ }^{1} \mathrm{H},{ }^{1} \mathrm{H}\right)=6.6 \mathrm{~Hz}, 1 \mathrm{H}\right.$ of $\mathrm{a}$, syn of $\left.\mathrm{C}_{3} H_{5}\right), 1.48$ $\left(\mathrm{s}, 9 \mathrm{H}\right.$ of $\mathbf{a}$ and $9 \mathrm{H}$ of $\left.\mathbf{b}, \mathrm{C}\left(\mathrm{CH}_{3}\right)_{3}\right), 1.06\left(\mathrm{~d},{ }^{3} J\left({ }^{1} \mathrm{H},{ }^{1} \mathrm{H}\right)=10.7 \mathrm{~Hz}\right.$, $1 \mathrm{H}$ of a, anti of $\left.\mathrm{C}_{3} \mathrm{H}_{5}\right), 0.85\left(\mathrm{~d},{ }^{3} J\left({ }^{1} \mathrm{H},{ }^{1} \mathrm{H}\right)=10.8 \mathrm{~Hz}, 1 \mathrm{H}\right.$ of a, anti of $\left.\mathrm{C}_{3} H_{5}\right), 0.78\left(\mathrm{~m}, 1 \mathrm{H}\right.$ of a, meso of $\left.\mathrm{C}_{3} H_{5}\right),-0.06\left(\mathrm{~d},{ }^{3} J\left({ }^{1} \mathrm{H},{ }^{1} \mathrm{H}\right)=\right.$ $9.6 \mathrm{~Hz}, 1 \mathrm{H}$ of $\mathbf{b}$, anti of $\left.\mathrm{C}_{3} H_{5}\right),-1.09\left(\mathrm{~d},{ }^{3} J\left({ }^{1} \mathrm{H},{ }^{1} \mathrm{H}\right)=9.7 \mathrm{~Hz}, 1 \mathrm{H}\right.$ of b, anti of $\left.\mathrm{C}_{3} H_{5}\right)$. IR (ATR, $\left.\mathrm{cm}^{-1}\right): 3372 \mathrm{~m}[\nu(\mathrm{NH})], 1932 \mathrm{vs}$ $\left[\nu_{\mathrm{a}}(\mathrm{CO})_{\mathrm{C} \equiv \mathrm{o}}\right], 1848 \mathrm{vs}\left[\nu_{\mathrm{s}}(\mathrm{CO})_{\mathrm{C} \equiv \mathrm{o}}\right], 1635 \mathrm{~s}\left[\nu(\mathrm{CO})_{\mathrm{C}=\mathrm{O}}\right]$. Raman (capillary, $\left.\mathrm{cm}^{-1}\right): 1931(6)\left[\nu_{\mathrm{a}}(\mathrm{CO})_{\mathrm{C} \equiv \mathrm{o}}\right], 1850(10)\left[\nu_{\mathrm{s}}(\mathrm{CO})_{\mathrm{C} \equiv \mathrm{o}}\right]$, $1637(4)\left[\nu(\mathrm{CO})_{\mathrm{C}=\mathrm{O}}\right]$. Single crystals of 20 suitable to X-ray diffraction analysis were prepared by slow evaporation of $\mathrm{MeOH}$ solution.

\section{Synthesis of $\left[\left(\eta^{3}-\mathrm{C}_{3} \mathrm{H}_{5}\right)\left\{\eta^{5}-1-(\mathrm{PhNHCO}) \mathrm{C}_{9} \mathrm{H}_{6}\right\} \mathrm{Mo}(\mathrm{CO})_{2}\right](21)$}

The steps of synthesis followed the procedure for compound 7. Reagents: 3-(PhNHCO) $\mathrm{C}_{9} \mathrm{H}_{7}(16 ; 0.47 \mathrm{~g}, 2 \mathrm{mmol}), 1.3 \mathrm{~mL}$ of ${ }^{n} \mathrm{BuLi}\left(1.6 \mathrm{~mol} \mathrm{~L}{ }^{-1}\right),\left[\left(\eta^{3}-\mathrm{C}_{3} \mathrm{H}_{5}\right) \mathrm{Mo}(\mathrm{CO})_{2}(\mathrm{NCMe})_{2} \mathrm{Cl}\right](6 ; 0.62 \mathrm{~g}$, $2 \mathrm{mmol})$. Yield: $0.45 \mathrm{~g}(1.05 \mathrm{mmol}, 53 \%)$. Yellow powder. $\mathrm{Mp}=$ $180{ }^{\circ} \mathrm{C}$ (dec.). Anal. Calc. for $\mathrm{C}_{21} \mathrm{H}_{17} \mathrm{MoNO}_{3}$ : C: 59.03; H: 4.01; H: 3.28. Found: C: $58.91 ; \mathrm{H}: 4.08$; H: $3.21 .{ }^{1} \mathrm{H}$ NMR (acetone- $d^{6}$, $400 \mathrm{MHz}, \delta$ ppm; 4:1 mixture of 21a $\left(\right.$ exo- $\left.\mathrm{C}_{3} \mathrm{H}_{5}\right)$ and 21b (endo$\left.\left.\mathrm{C}_{3} \mathrm{H}_{5}\right)\right): 9.24(1 \mathrm{H}$ of $\mathbf{a}, \mathrm{NH}), 9.13(1 \mathrm{H}$ of $\mathbf{b}, \mathrm{NH}), 8.16-6.95(\mathrm{~m}, 9 \mathrm{H}$ of a and $9 \mathrm{H}$ of $\mathbf{b}, \mathrm{H}^{4-7}$ of $\left.\mathrm{C}_{9} H_{6}, \mathrm{C}_{6} H_{5}\right), 6.71\left(\mathrm{~d},{ }^{3} J\left({ }^{1} \mathrm{H},{ }^{1} \mathrm{H}\right)=3.2 \mathrm{~Hz}\right.$, $1 \mathrm{H}$ of $\mathbf{a}, \mathrm{H}^{2,3}$ of $\mathrm{C}_{9} H_{6}$ ), 6.61 (s-br, $1 \mathrm{H}$ of $\mathbf{b}, \mathrm{H}^{2,3}$ of $\mathrm{C}_{9} H_{6}$ ), 6.32 $\left(\mathrm{d},{ }^{3} J\left({ }^{1} \mathrm{H},{ }^{1} \mathrm{H}\right)=3.2 \mathrm{~Hz}, 1 \mathrm{H}\right.$ of $\mathbf{a}, \mathrm{H}^{2,3}$ of $\left.\mathrm{C}_{9} H_{6}\right), 6.28$ (s-br, $1 \mathrm{H}$ of $\mathbf{b}$, $\mathrm{H}^{2,3}$ of $\mathrm{C}_{9} H_{6}$ ), 3.50 (s-br, $1 \mathrm{H}$ of $\mathbf{b}, \mathrm{C}_{3} H_{5}$ ), 3.36 (s-br, $2 \mathrm{H}$ of $\mathbf{b}$, $\left.\mathrm{C}_{3} H_{5}\right), 2.36\left(\mathrm{~d},{ }^{3} J\left({ }^{1} \mathrm{H},{ }^{1} \mathrm{H}\right)=7.2 \mathrm{~Hz}, 1 \mathrm{H}\right.$ of a, syn of $\left.\mathrm{C}_{3} H_{5}\right), 2.14$ $\left(\mathrm{d},{ }^{3} J\left({ }^{1} \mathrm{H},{ }^{1} \mathrm{H}\right)=7.0 \mathrm{~Hz}, 1 \mathrm{H}\right.$ of $\mathbf{a}$, syn of $\left.\mathrm{C}_{3} H_{5}\right), 1.15(\mathrm{~m}, 1 \mathrm{H}$ of $\mathbf{a}$, meso of $\left.\mathrm{C}_{3} \mathrm{H}_{5}\right), 0.99\left(\mathrm{~d},{ }^{3} \mathrm{~J}\left({ }^{1} \mathrm{H},{ }^{1} \mathrm{H}\right)=11.3 \mathrm{~Hz}, 1 \mathrm{H}\right.$ of $\mathrm{a}$, anti of $\left.\mathrm{C}_{3} H_{5}\right), 0.82\left(\mathrm{~d},{ }^{3} J\left({ }^{1} \mathrm{H},{ }^{1} \mathrm{H}\right)=11.4 \mathrm{~Hz}, 1 \mathrm{H}\right.$ of $\mathrm{a}$, anti of $\left.\mathrm{C}_{3} H_{5}\right),-0.09$ $\left(\mathrm{d},{ }^{3} J\left({ }^{1} \mathrm{H},{ }^{1} \mathrm{H}\right)=10.7 \mathrm{~Hz}, 1 \mathrm{H}\right.$ of $\mathbf{b}$, anti of $\left.\mathrm{C}_{3} H_{5}\right),-0.94$ $\left(\mathrm{d},{ }^{3} J\left({ }^{1} \mathrm{H},{ }^{1} \mathrm{H}\right)=10.8 \mathrm{~Hz}, 1 \mathrm{H}\right.$ of $\mathbf{b}$, anti of $\left.\mathrm{C}_{3} \mathrm{H}_{5}\right)$. IR (ATR, $\left.\mathrm{cm}^{-1}\right)$ : $3307 \mathrm{~m}[\nu(\mathrm{NH})], 1932 \mathrm{vs}\left[\nu_{\mathrm{a}}(\mathrm{CO})_{\mathrm{C} \equiv \mathrm{o}}\right], 1859 \mathrm{vs}\left[\nu_{\mathrm{s}}(\mathrm{CO})_{\mathrm{C} \equiv \mathrm{o}}\right], 1633 \mathrm{~s}$ $\left[\nu(\mathrm{CO})_{\mathrm{C}=\mathrm{O}}\right]$. Raman (capillary, $\left.\mathrm{cm}^{-1}\right): 1933(5) \quad\left[\nu_{\mathrm{a}}(\mathrm{CO})_{\mathrm{C} \equiv \mathrm{O}}\right]$, $1866(9)\left[\nu_{\mathrm{s}}(\mathrm{CO})_{\mathrm{C} \equiv \mathrm{O}}\right], 1635(8)\left[\nu(\mathrm{CO})_{\mathrm{C}=\mathrm{O}}\right]$.

\section{Synthesis of $\left[\left(\eta^{3}-\mathrm{C}_{3} \mathrm{H}_{5}\right)\left\{\eta^{5}-1-\left(4-\mathrm{ClC}_{6} \mathrm{H}_{4} \mathrm{NHCO}\right) \mathrm{C}_{9} \mathrm{H}_{6}\right\} \mathrm{Mo}(\mathrm{CO})_{2}\right]$} (22)

The steps of synthesis followed the procedure for compound 7 . Reagents: 3-(4-ClC $\left.{ }_{6} \mathrm{H}_{4} \mathrm{NHCO}\right) \mathrm{C}_{9} \mathrm{H}_{7}(19 ; 0.54 \mathrm{~g}, 2 \mathrm{mmol}), 1.3 \mathrm{~mL}$ of ${ }^{n} \mathrm{BuLi}\left(1.6 \mathrm{~mol} \mathrm{~L}^{-1}\right),\left[\left(\eta^{3}-\mathrm{C}_{3} \mathrm{H}_{5}\right) \mathrm{Mo}(\mathrm{CO})_{2}(\mathrm{NCMe})_{2} \mathrm{Cl}\right](6 ; 0.62 \mathrm{~g}$, $2 \mathrm{mmol})$. Yield: $0.55 \mathrm{~g}$ (1.19 mmol, 60\%). Yellow powder. $\mathrm{Mp}=$ $185{ }^{\circ} \mathrm{C}$ (dec.). Anal. Calc. for $\mathrm{C}_{21} \mathrm{H}_{16} \mathrm{ClMoNO}_{3}$ : C: 54.62; H: 3.49; $\mathrm{N}: 3.03$. Found: $\mathrm{C}: 54.70 ; \mathrm{H}: 3.52 ; \mathrm{N}: 3.08 .{ }^{1} \mathrm{H} \mathrm{NMR}\left(\mathrm{CDCl}_{3}\right.$, $400 \mathrm{MHz}, \delta \mathrm{ppm} ; 3: 1$ mixture of 23a $\left(\right.$ exo- $\left.\mathrm{C}_{3} \mathrm{H}_{5}\right)$ and $23 \mathbf{b}$ (endo$\left.\left.\mathrm{C}_{3} \mathrm{H}_{5}\right)\right): 7.98\left(\mathrm{~d},{ }^{3} J\left({ }^{1} \mathrm{H},{ }^{1} \mathrm{H}\right)=8.8 \mathrm{~Hz}, 1 \mathrm{H}\right.$ of $\mathbf{a}$ and $1 \mathrm{H}$ of $\mathbf{b}, \mathrm{H}^{4,7}$ of $\left.\mathrm{C}_{9} H_{6}\right), 7.60-7.05\left(\mathrm{~m}, 8 \mathrm{H}\right.$ of $\mathbf{a}$ and $8 \mathrm{H}$ of $\mathbf{b}, \mathrm{H}^{4-7}$ of $\mathrm{C}_{9} H_{6}, \mathrm{C}_{6} H_{4}$, $\mathrm{NH}), 6.10\left(\mathrm{~d},{ }^{3} J\left({ }^{1} \mathrm{H},{ }^{1} \mathrm{H}\right)=2.9 \mathrm{~Hz}, 1 \mathrm{H}\right.$ of $\mathbf{a}, \mathrm{H}^{2,3}$ of $\left.\mathrm{C}_{9} H_{6}\right), 6.05$ $\left(\mathrm{d},{ }^{3} J\left({ }^{1} \mathrm{H},{ }^{1} \mathrm{H}\right)=2.9 \mathrm{~Hz}, 1 \mathrm{H}\right.$ of $\mathbf{a}, \mathrm{H}^{2,3}$ of $\left.\mathrm{C}_{9} H_{6}\right), 6.03\left(\mathrm{~d},{ }^{3} J\left({ }^{1} \mathrm{H},{ }^{1} \mathrm{H}\right)=\right.$ $2.8 \mathrm{~Hz}, 1 \mathrm{H}$ of $\mathbf{b}, \mathrm{H}^{2,3}$ of $\left.\mathrm{C}_{9} H_{6}\right), 5.98\left(\mathrm{~d},{ }^{3} J\left({ }^{1} \mathrm{H},{ }^{1} \mathrm{H}\right)=2.8 \mathrm{~Hz}, 1 \mathrm{H}\right.$ of $\mathbf{b}, \mathrm{H}^{2,3}$ of $\mathrm{C}_{9} H_{6}$ ), 3.62 (s-br, $1 \mathrm{H}$ of $\mathbf{b}, \mathrm{C}_{3} H_{5}$ ), 3.39 (s-br, $2 \mathrm{H}$ of $\mathbf{b}$, $\left.\mathrm{C}_{3} H_{5}\right), 2.47\left(\mathrm{~d},{ }^{3} J\left({ }^{1} \mathrm{H},{ }^{1} \mathrm{H}\right)=6.7 \mathrm{~Hz}, 1 \mathrm{H}\right.$ of a, syn of $\left.\mathrm{C}_{3} H_{5}\right), 2.21$ $\left(\mathrm{d},{ }^{3} J\left({ }^{1} \mathrm{H},{ }^{1} \mathrm{H}\right)=6.7 \mathrm{~Hz}, 1 \mathrm{H}\right.$ of $\mathbf{a}$, syn of $\left.\mathrm{C}_{3} H_{5}\right), 1.14\left(\mathrm{~d},{ }^{3} J\left({ }^{1} \mathrm{H},{ }^{1} \mathrm{H}\right)=\right.$ $11.1 \mathrm{~Hz}, 1 \mathrm{H}$ of a, anti of $\left.\mathrm{C}_{3} H_{5}\right), 0.90\left(\mathrm{~d},{ }^{3} J\left({ }^{1} \mathrm{H},{ }^{1} \mathrm{H}\right)=11.6 \mathrm{~Hz}, 1 \mathrm{H}\right.$ of a, anti of $\left.\mathrm{C}_{3} \mathrm{H}_{5}\right), 0.64\left(\mathrm{~m}, 1 \mathrm{H}\right.$ of a, meso of $\left.\mathrm{C}_{3} \mathrm{H}_{5}\right),-0.06$ $\left(\mathrm{d},{ }^{3} J\left({ }^{1} \mathrm{H},{ }^{1} \mathrm{H}\right)=9.8 \mathrm{~Hz}, 1 \mathrm{H}\right.$ of $\mathbf{b}$, anti of $\left.\mathrm{C}_{3} \mathrm{H}_{5}\right),-1.00\left(\mathrm{~d},{ }^{3} J\left({ }^{1} \mathrm{H},{ }^{1} \mathrm{H}\right)\right.$ $=9.6 \mathrm{~Hz}, 1 \mathrm{H}$ of $\mathbf{b}$, anti of $\left.\mathrm{C}_{3} H_{5}\right)$. IR (ATR, $\left.\mathrm{cm}^{-1}\right): 3424 \mathrm{~m}[\nu(\mathrm{NH})]$, $1940 \mathrm{vs}\left[\nu_{\mathrm{a}}(\mathrm{CO})_{\mathrm{C} \equiv \mathrm{o}}\right], 1852 \mathrm{vs}\left[\nu_{\mathrm{s}}(\mathrm{CO})_{\mathrm{C} \equiv \mathrm{o}}\right], 1667 \mathrm{~s}\left[\nu(\mathrm{CO})_{\mathrm{C}=\mathrm{O}}\right]$. Raman (capillary, $\left.\mathrm{cm}^{-1}\right): \quad 1948(3) \quad\left[\nu_{\mathrm{a}}(\mathrm{CO})_{\mathrm{C} \equiv \mathrm{o}}\right], \quad 1848(8)$ $\left[\nu_{\mathrm{s}}(\mathrm{CO})_{\mathrm{C} \equiv \mathrm{O}}\right], 1664(10)\left[\nu(\mathrm{CO})_{\mathrm{C}=\mathrm{O}}\right]$. Single crystals of $23 \cdot \mathrm{MeOH}$ suitable to X-ray diffraction analysis were prepared by slow evaporation of $\mathrm{MeOH}$ solution.

\section{X-ray crystallography}

The X-ray data for the crystals of compounds 7, 12, 13, 14, 20 and 22. $\mathrm{MeOH}$ were obtained at $150 \mathrm{~K}$ using an Oxford Cryostream 
low-temperature device on a Nonius KappaCCD diffractometer with Mo $K \alpha$ radiation $(\lambda=0.71073 \AA)$ and a graphite monochromator. Data reductions were performed using DENZO$\mathrm{SMN}^{26}$ The structures were solved by direct methods (Sir92) ${ }^{27}$ and refined by full-matrix least squares based on $F^{2}$ (SHELXL). ${ }^{28}$ Hydrogen atoms were mostly localized on a difference Fourier map. However, to ensure uniformity of the treatment of the crystals, all hydrogen atoms were recalculated into idealized positions (riding model) and assigned temperature factors $U_{\text {iso }}(\mathrm{H})=1.2\left[U_{\text {eq }}(\right.$ pivot atom $\left.)\right]$ or $1.5 U_{\text {eq }}$ for the methyl moiety with $\mathrm{C}-\mathrm{H}=0.96,0.97$, and $0.93 \AA$ for methyl, methylene, and hydrogen atoms in aromatic rings or the allyl moiety, respectively. The structure of 7 contains a disorder of the allyl group which, is positionaly disordered on one of the carbon atoms (C13) and splits into two positions with an occupancy of about $7: 3$; this disorder was treated using SHELXL software. ${ }^{28}$

\section{Acknowledgements}

The NMR spectrometers at ITQB are part of the National NMR Facility, supported by Fundação para a Ciência e a Tecnologia (RECI/BBB-BQB/0230/2012). We thank University of Pardubice for the Erasmus mobility fellowship (Erasmus+ 2014/2015).

\section{References}

1 (a) T. Liu, X. Wang, C. Hoffmann, D. L. DuBois and M. R. Bullock, Angew. Chem., Int. Ed., 2014, 53, 5300-5304; (b) M. Fang, E. S. Wiedner, W. G. Dougherty, W. S. Kassel, T. Liu, D. L. DuBois and M. R. Bullock, Organometallics, 2014, 33, 5820-5833; (c) X. Wang, D. Li, W. Deuther-Conrad, J. Lu, Y. Xie, B. Jia, M. Cui, J. Steinbach, P. Brust, B. Liu and H. Jia, J. Med. Chem., 2014, 57, 7113-7125; (d) S. Sulieman, D. Can, J. Mertens, H. W. P. N'Dongo, Y. Liu, P. Schmutz, M. Bauwens, B. Spingler and R. Alberto, Organometallics, 2012, 31, 6880-6886; (e) D. Can, B. Spingler, P. Schmutz, F. Mendes, P. Raposinho, C. Fernandes, F. Carta, A. Innocenti, I. Santos, C. T. Supuran and R. Alberto, Angew. Chem., Int. Ed., 2012, 51, 3354-3357; $(f)$ E. Hillard, A. Vessieres, L. Thouin, G. Jaouen and C. Amatore, Angew. Chem., Int. Ed., 2006, 45, 285-290; (g) C. Ornelas, New J. Chem., 2011, 35, 1973-1985; (h) J. H. Scarborough, K. Brusoski, S. Brewer, S. Rodich, K. S. Chatley, T. Nguyen and K. N. Green, Organometallics, 2015, 34, 918-925; (i) Y. Yan, T. M. Deaton, J. Zhang, H. He, J. Hayat, P. Pageni, K. Matyjaszewski and C. Tang, Macromolecules, 2015, 48, 1644-1650; (j) K. Strohfeldt and M. Tacke, Chem. Soc. Rev., 2008, 37, 1174-1187; (k) G. Erker, Dalton Trans., 2011, 40, 7475-7483; (l) S. S. Braga and A. M. S. Silva, Organometallics, 2013, 32, 5626-5639; (m) S. Ursillo, D. Can, H. W. P. N'Dongo, P. Schmutz, B. Spingler and R. Alberto, Organometallics, 2014, 33, 6945-6952.

2 P. J. Chirik, Organometallics, 2010, 29, 1500-1517.

3 S. Manzini, J. A. Fernández-Salas and S. P. Nolan, Acc. Chem. Res., 2014, 47, 3089-3101.
4 (a) J. M. O'Connor and C. P. Casey, Chem. Rev., 1987, 87, 307-318; (b) H. Bang, T. J. Lynch and F. Basolo, Organometallics, 1992, 11, 40-48; (c) C. C. Romão, Appl. Organomet. Chem., 2000, 14, 539-548.

5 (a) M. J. Calhorda, C. C. Romão and L. F. Veiros, Chem. Eur. J., 2002, 8, 868-875; (b) M. J. Calhorda and L. F. Veiros, Coord. Chem. Rev., 1999, 185-186, 37-51.

6 G. P. McGovern, F. Hung-Low, J. W. Tye and C. A. Bradley, Organometallics, 2012, 31, 3865-3879.

7 J. Honzíček, C. C. Romão, M. J. Calhorda, A. Mukhopadhyay, J. Vinklárek and Z. Padělková, Organometallics, 2011, 30, 717-725.

8 P. A. Deck, W. F. Jackson and F. R. Fronczek, Organometallics, 1996, 15, 5287-5291.

9 P. A. Deck and F. R. Fronczek, Organometallics, 2000, 19, 327-333.

10 K. Sünkel, S. Weigand, A. Hoffmann, S. Blomeyer, C. G. Reuter, Y. V. Vishnevskiy and N. W. Mitzel, J. Am. Chem. Soc., 2015, 137, 126-129.

11 P. J. Graham, V. Lindsey, G. W. Parshall, M. L. Peterson and G. M. Whitman, J. Am. Chem. Soc., 1957, 79, 3416-3419.

12 W. P. Hart, D. W. Macomber and M. D. Rausch, J. Am. Chem. Soc., 1980, 102, 1196-1198.

13 (a) L. Busetto, C. Cassani, V. Zanotti, V. G. Albano and P. Sabatino, Organometallics, 2001, 20, 282-288; (b) J. Honzíček, A. Mukhopadhyay, T. S. Silva, M. J. Romão and C. C. Romão, Organometallics, 2009, 28, 2871-2879; (c) A. R. Petrov, K. Jess, M. Freytag, P. G. Jones and M. Tamm, Organometallics, 2013, 32, 5946-5954.

14 (a) M. Oberhoff, L. Duda, J. Karl, R. Mohr, G. Erker, R. Fröhlich and M. Grehl, Organometallics, 1996, 15, 4005-4011; (b) K. Klaß, R. Fröhlich and G. Erker, J. Chem. Soc., Dalton Trans., 1999, 4457-4461; (c) H. Goitia, Y. Nieto, M. D. Villacampa, C. Kasper, A. Laguna and M. C. Gimeno, Organometallics, 2013, 32, 6069-6078.

15 J. Honzíček, P. Kratochvíl, J. Vinklárek, A. Eisner and Z. Padělková, Organometallics, 2012, 31, 2193-2202.

16 J. Schejbal, J. Honzíček, J. Vinklárek, M. Erben and Z. Růžičková, Eur. J. Inorg. Chem., 2014, 5895-5907.

17 (a) W. E. Noland, L. L. Landucci and V. Kameswaran, J. Org. Chem., 1980, 45, 3456-3461; (b) T. Takahashi, H. Kameda, T. Kamei and M. Ishizaki, J. Fluorine Chem., 2006, 127, 760-768; (c) G. R. Clemo, L. H. Groves, L. Munday and G. A. Swan, J. Chem. Soc., 1951, 863-867.

18 J. W. Faller, C. C. Chen, M. J. Mattina and A. Jakubowski, J. Organomet. Chem., 1973, 52, 361-386.

19 (a) P. Neves, C. C. L. Pereira, F. A. A. Paz, S. Gago, M. Pillinger, C. M. Silva, A. A. Valente, C. C. Romão and I. S. Gonçalves, J. Organomet. Chem., 2010, 695, 2311-2319; (b) F. Pammer, Y. Sun and W. R. Thiel, Organometallics, 2008, 27, 1015-1018; (c) J. Honzíček, J. Vinklárek, M. Erben, Z. Padělková, L. Šebestová and M. Řezáčová, J. Organomet. Chem., 2014, 749, 387-393; (d) J. Honzíček, J. Vinklárek, Z. Padělková, L. Šebestová, K. Foltánová and M. Řezáčová, J. Organomet. Chem., 2012, 716, 258-268.

20 J. Honzíček, J. Vinklárek, M. Erben, J. Lodinský, L. Dostál and Z. Padělková, Organometallics, 2013, 32, 3502-3511. 
21 J. Pietruszka, R. C. Simon, F. Kruska and M. Braun, Eur. J. Org. Chem., 2009, 6217-6224.

22 J. W. Faller, R. H. Crabtree and A. Habib, Organometallics, 1985, 4, 929-935.

23 M. G. B. Drew, V. Félix, I. S. Gonçalves, C. C. Romão and B. Royo, Organometallics, 1998, 17, 5782-5788.

24 J. R. Ascenso, C. G. de Azevedo, I. S. Gonçalves, E. Herdtweck, D. S. Moreno, M. Pessanha and C. C. Romão, Organometallics, 1995, 14, 3901-3919.
25 W. L. F. Armarego and D. D. Perrin, Purification of Laboratory Chemicals, Oxford, 1996.

26 Z. Otwinowski and W. Minor, Methods Enzymol., 1997, 276, 307-326.

27 A. Altomare, G. Cascarano, C. Giacovazzo, A. Guagliardi, M. C. Burla, G. Polidori and M. Camalli, J. Appl. Crystallogr., 1994, 27, 435-436.

28 G. M. Sheldrick, SHELXL97, University of Göttingen, Germany, 2008. 